\title{
Lexical frequency and sentence context influence the brain's response to single words
}

\section{Lexical frequency in sentence context}

\author{
Eleanor Huizeling ${ }^{1 *}$, Sophie Arana ${ }^{1,2}$, Peter Hagoort ${ }^{1,2}$, Jan Mathijs Schoffelen ${ }^{2}$ \\ ${ }^{1}$ Max Planck Institute for Psycholinguistics, Nijmegen, The Netherlands \\ ${ }^{2}$ Donders Institute for Brain, Cognition and Behaviour, Nijmegen, The Netherlands \\ *Correspondence: \\ Dr Eleanor Huizeling \\ eleanor.huizeling@mpi.nl \\ Max Planck Institute for Psycholinguistics, Wundtlaan 1, 6525XD, Nijmegen, The Netherlands.
}

Acknowledgements:

We would like to thank Alessandro Lopopolo for computing the corpus-derived lexical characteristics of lexical frequency, surprisal and entropy for the current stimulus set.

Authors report no conflict of interest.

This work was supported by The Netherlands Organisation for Scientific Research (NWO Vidi: 864.14.011). 


\section{$1 \quad \underline{\text { Abstract }}$}

2 Typical adults read remarkably quickly. Such fast reading is facilitated by brain processes that are

3 sensitive to both word frequency and contextual constraints. It is debated as to whether these

4 attributes have additive or interactive effects on language processing in the brain. We investigated

5 this issue by analysing existing magnetoencephalography data from 99 participants reading intact

6 and scrambled sentences. Using a cross-validated model comparison scheme, we found that lexical

7 frequency predicted the word-by-word elicited MEG signal in a widespread cortical network,

8 irrespective of sentential context. In contrast, index (ordinal word position) was more strongly

9 encoded in sentence words, in left front-temporal areas. This confirms that frequency influences

10 word processing independently of predictability, and that contextual constraints affect word-by-

11 word brain responses. With a conservative multiple comparisons correction, only the interaction

12 between lexical frequency and surprisal survived, in anterior temporal and frontal cortex, and not

13 between lexical frequency and entropy, nor between lexical frequency and index. However,

14 interestingly, the uncorrected index*frequency interaction revealed an effect in left frontal and

15 temporal cortex that reversed in time and space for intact compared to scrambled sentences.

16 Finally, we provide evidence to suggest that, in sentences, lexical frequency and predictability may

17 independently influence early $(<150 \mathrm{~ms})$ and late stages of word processing, but also interact during

18 late stages of word processing (>150-250ms), thus helping to converge previous contradictory eye-

19 tracking and electrophysiological literature. Current neuro-cognitive models of reading would

20 benefit from accounting for these differing effects of lexical frequency and predictability on

21 different stages of word processing. 


\section{Introduction}

24 When reading a text, the reader's brain is capable of rapidly extracting meaning from the structured

25 sequence of individual words. In order to achieve its remarkable efficiency in processing, the brain

26 network for language not only extracts, and actively uses, lexical properties of the individual

27 words, but is also greatly influenced by the context in which those words occur. On the one hand,

28 for instance, words that maintain a highly frequent occurrence in day-to-day language use are

29 processed faster and with less effort than words that occur less frequently (Calvo \& Meseguer,

30 2002; Inhoff \& Rayner, 1986; Rayner \& Duffy, 1986; Rubenstein, Garfield, \& Millikan, 1970).

31 On the other hand, as a linguistic expression unfolds, the previously read input provides the brain

32 with a constraining semantic and syntactic context, which may allow for predictions to be made

33 about the upcoming word. This results in measurable effects at fast timescales, in response times

34 (Staub, Grant, Astheimer, \& Cohen, 2015), and in both electrophysiological (Van Petten \& Kutas,

35 1990) and eye movement signals (Calvo \& Meseguer, 2002).

36 Typical adult readers effortlessly process an average of 238 words per minute (Brysbaert, 2019),

37 fixating on each word for an average of only 235ms (Rayner, 1986). The brain's rapid word

38 processing has been shown to be facilitated when the word frequently occurs within a given

39 language (i.e. has a high lexical frequency). Compared to low frequency words, high frequency

40 words are fixated for shorter durations during reading (Calvo \& Meseguer, 2002; Inhoff \& Rayner,

41 1986; Rayner \& Duffy, 1986), are responded to faster in lexical decision tasks (Rubenstein et al.,

42 1970), and produce smaller electrophysiological (Smith \& Halgren, 1987; Van Petten \& Kutas,

43 1990) and hemodynamic responses (Chee, Hon, Caplan, Lee, \& Goh, 2002). Although the specific

44 temporal and spatial dynamics of electrophysiological frequency effects may be sensitive to task 
context (Chen, Davis, Pulvermüller, \& Hauk, 2015; Strijkers, Bertrand, \& Grainger, 2015), overall,

46 it seems that processing of high frequency words is less effortful than low frequency words.

47 The prediction of upcoming sentential content is another mechanism that seems to facilitate the

48 remarkable speed of sentence reading. There is now ample evidence that one is able to predict

49 upcoming linguistic input, although whether this is to the level of semantics, syntactic content or

50 the word form is still debated (Pickering \& Gambi, 2018). Regardless of the level at which

51 prediction takes place, highly predictable words seem to be processed faster than unpredictable

52 words, reflected in shorter fixation durations (Calvo \& Meseguer, 2002; Rayner \& Well, 1996)

53 and smaller N400 responses (Van Petten \& Kutas, 1990). The N400 is an electrophysiological

54 marker of semantic processing, which occurs between 200-600ms at a centro-parietal topography,

55 and is thought to reflect either the integration and unification of semantic information (Hagoort,

56 Baggio, \& Willems, 2009; Kutas \& Federmeier, 2011) or conceptual (or possibly lexical) pre-

57 activation (Lau \& Namyst, 2019; Lau, Phillips, \& Poeppel, 2008). A larger N400 response is

58 observed when the integration of semantic information is more difficult, or in the absence of

59 conceptual/lexical pre-activation, for example when the word is less predictable.

60 There is increasing agreement that there are two mechanisms through which prediction can take

61 place. Firstly, through a fast, effortless and automatic mechanism, in which activity spreads to

62 associated features, or, secondly, through a higher level mechanism, in which world knowledge

63 and the surrounding context are combined to form predictions (Huettig, 2015; Pickering \& Gambi,

64 2018). Lexical frequency could therefore influence the automatic, bottom-up prediction

65 mechanism, where activation thresholds are lower for high compared to low frequency words. In

66 contrast, effects of the semantic and syntactic constraints, provided by the context that a word is

67 presented in, may reflect a prediction mechanism that relies on the top-down flow of information 
68 from strong priors. For example, as semantic context increases as the sentence unfolds a stronger

69 foundation on which to base predictions is provided. In this study, we follow earlier approaches in

70 using ordinal word position in a sentence (or index) to roughly quantify context. Indeed, the N400

71 has been shown to decrease with both increased lexical frequency and increased index

72 (Dambacher, Kliegl, Hofmann, \& Jacobs, 2006; Payne, Lee, \& Federmeier, 2015; Van Petten \&

73 Kutas, 1990), which suggests that word integration becomes easier as each of these factors

74 increase.

75 A recurring finding in the literature is that there is an interaction between effects of increased

76 predictability and lexical frequency on the N400, where the effect of word frequency on the N400

77 amplitude during word processing is greatly diminished or disappears with increased context or

78 predictability (Alday, Schlesewsky, \& Bornkessel-Schlesewsky, 2017; Dambacher et al., 2006;

79 Payne et al., 2015; Sereno, Hand, Shahid, Mackenzie, \& Leuthold, 2019; Van Petten \& Kutas,

80 1990). Similar interactions have also been observed at earlier time windows (Dambacher et al.,

81 2012; Sereno, Brewer, \& O'Donnell, 2003; Sereno et al., 2019) and with functional near-infrared

82 spectroscopy (fNIRS; Hofmann et al., 2014). In an MEG study, Fruchter, Linzen, Westerlund, and

83 Marantz (2015) additionally found word frequency and predictability to interact in the left MTG,

84 in time windows both preceding and succeeding the predictable word onset. Overall, these findings

85 demonstrate that the interaction between lexical frequency and increased context is a robust and

86 well replicated finding, which reflects both the reduced influence of lexical frequency on word

87 processing with increased context, as well as a greater benefit of predictability for processing low

88 compared to high frequency words.

89 The reduced effect of lexical frequency on word processing with increased context has lead authors

90 to conclude that lexical frequency merely reflects a bottom-up, baseline level of expectation that 
91 is soon overridden with top-down information in the presence of context (Kretzschmar,

92 Schlesewsky, \& Staub, 2015). However, there is a well-documented discrepancy between the

93 aforementioned electrophysiological literature and the eye-tracking literature as to whether

94 frequency and predictability indeed have an interactive effect on word processing, or whether

95 effects are additive (Kretzschmar et al., 2015). In contrast to the findings of the N400 literature,

96 recording participants' eye gaze during reading has consistently demonstrated an additive effect

97 of lexical frequency and predictability on fixation durations. Fixation durations are longer for

98 highly predictable low frequency words than highly predictable high frequency words, and again

99 longer for unpredictable low frequency words (Kennedy, Pynte, Murray, \& Paul, 2013;

100 Kretzschmar et al., 2015; Staub, 2015; Staub \& Benatar, 2013). One explanation for these

101 contradictory findings is that lexical frequency and prediction have separate additive effects during

102 early processing stages (Sereno et al., 2019; Staub \& Goddard, 2019), for example during

103 sublexical orthographic processing, morphological decomposition or lexical retrieval, but that

104 frequency effects are not present with increased context during later semantic processing and

105 integration.

106 1.1. The current work

107 Considering the aforementioned ambiguity in the theoretical understanding of how lexical

108 frequency influences subsequent processing, specifically in the light of additional context-based

109 predictability, the current work performed a novel analysis on an existing dataset, with the aim to

110 dissociate lexical frequency effects from predictability effects. Although previous work has sought

111 to define when frequency and predictability interact, less attention has been invested into

112 examining the spatiotemporal dynamics of this interaction (although, see the exploratory analysis

113 in Fruchter et al., 2015 for an exception). We aimed to determine at which time points and in which 
114 locations lexical frequency and predictability independently influence word processing, and at

115 which points they interact, thereby providing valuable information for models of word reading.

116 Staub and Goddard (2019) recently highlighted that current models of word reading, such as the

117 E-Z reader (Reichle, Rayner, \& Pollatsek, 2003) and SWIFT (Engbert, Nuthmann, Richter, \&

118 Kliegl, 2005), do not yet completely account for effects of predictability and invalid previews on

119 fixation durations. Considering the complex effects lexical attributes have on the neural processing

120 of language, a comprehensive account of word reading could benefit from improving upon both

121 the temporal and spatial resolution of previous work.

122 Specifically, we used the Mother of all Unification Studies (MOUS; Schoffelen et al., 2019), a

123 large sample size open-access dataset of 102 participants in which magnetoencephalography

124 (MEG) was recorded while they read intact sentences and scrambled sentences. Improving upon

125 previous electroencephalography (EEG), functional magnetic resonance imaging (fMRI) and

126 fNIRS research, MEG provides both the temporal and spatial resolution to detect subtle and fine-

127 grained differences in the extent that lexical frequency and predictability are encoded in the MEG

128 signal after word-onset, which could have previously been lost by averaging over time and space.

129 Distinct from most previous work, with respect to the analysis, we exploited the word-by-word

130 variability in the MEG signal, which is often lost through averaging across words of the same

131 experimental condition. Specifically, we used multiset canonical correlation analysis (MCCA) to

132 boost the stimulus-specific signal (Arana, Marquand, Hultén, Hagoort, \& Schoffelen, 2020), and

133 performed detailed cross-validated single-trial encoding model analysis, using regression models

134 that quantified the degree to which lexical frequency and various measures of predictability are 135 encoded in the MEG signal. 
136 To investigate the extent that context influences effects of lexical frequency on word processing,

137 we first compared sentences and scrambled sentences as to the amount of variance in the ongoing

138 brain signal explained by lexical frequency. The scrambled sentences were created by randomly

139 shuffling the order of the words in the intact sentences, and therefore matched the intact sentences

140 word-for-word, differing only in the order that words were presented in. This meant that the two

141 conditions (intact/scrambled) differed only in the presence/absence, respectively, of the build-up

142 of a rich sentence context. Although some degree of sparse combinatorial processing may have

143 been possible at the semantic level in the scrambled sentences, the ability to derive a coherent

144 sentence level context and produce top-down driven predictions was possible only in the sentences.

145 In addition to the level of sentential context provided by the presence/absence of syntax, we

146 approximately quantified context with the ordinal word position in the sentence (index), consistent

147 with previous approaches (Dambacher et al., 2006; Payne et al., 2015; Van Petten \& Kutas, 1990).

148 Index captures the incremental build-up of the entire sentence context. Moreover, as context

149 increases with increased word position, predictability is expected to increase with increased

150 context (for a similar argument, see Levy, 2008; Schuster, Hawelka, Himmelstoss, Richlan, \&

151 Hutzler, 2020). Thus, effects of index were expected to differ in intact compared to scrambled

152 sentences. Whereas index provided a correlate of predictability that encompassed the entire

153 sentence context, surprisal and entropy were used to provide measures of local predictability

154 (acquired from a trained tri-gram model). Specifically, surprisal quantifies how unexpected the

155 current word is, and entropy represents the uncertainty of the upcoming word. Effects of surprisal

156 and entropy were compared across intact and scrambled sentence conditions, in order to identify

157 effects related to higher level predictive processes, which were only possible in the sentence

158 condition. We investigated the interaction between lexical frequency and each variable quantifying 
159 different degrees of predictability (index, surprisal and entropy). Lexical frequency (rather than

160 lemma frequency) was chosen to quantify word frequency effects, in order to remain consistent

161 with most previous reports (Alday et al., 2017; Dambacher et al., 2006; Payne et al., 2015; Sereno

162 et al., 2019; Van Petten \& Kutas, 1990). As effects of lexical frequency and predictability on the

163 electrophysiological response have been shown to interact with word length (Penolazzi, Hauk, \&

164 Pulvermuller, 2007), word length was added as a control predictor to all models. Due to

165 fundamental differences in the properties of content words (nouns, adjectives, verbs) and function

166 words (determiners, prepositions, pronouns, conjunctions), for example in their frequency, length

167 and semantic richness, they were analysed separately (see Matchin, Brodbeck, Hammerly, \& Lau,

1682019 for a similar approach). Only content words were included in the analysis here.

169 Although Fruchter et al. (2015) previously studied the spatiotemporal effects of a similar 170 interaction using MEG, our study differed from theirs in a number of ways, providing additional

171 contributions to the field. Firstly, in contrast to Fruchter et al. (2015), our stimuli were not designed

172 to be highly predictable, and were not limited to measuring the response to adjective-noun pairs

173 such as "stainless steel", selected based on co-occurrence statistics. We therefore investigated the

174 spatiotemporal dynamics of the interaction with a richer stimulus set, which is arguably closer to

175 the linguistic content one would read in everyday situations, where sentences are not always highly

176 predictable, and also depend upon integrating world knowledge. The prediction of frequently co-

177 occurring words would arguably depend on different processing mechanisms (e.g. priming)

178 compared to forming predictions based on the build-up of context constraints (Huettig, 2015;

179 Pickering \& Gambi, 2018). Secondly, we investigated the effect of the interaction over time and

180 space, rather than averaging over time windows or using single regions-of-interests (ROIs).

181 Although Fruchter et al. (2015) also presented the spatiotemporal dynamics of the interaction, this 
182 was in an exploratory analysis that requires replication. Their primary analyses averaged over

183 longer time windows and were restricted to several ROIs. Furthermore, in their exploratory

184 spatiotemporal analysis, the authors averaged over $100 \mathrm{~ms}$ time windows. We here provide finer

185 grained information about the spatiotemporal dynamics of the interaction between lexical

186 frequency and context. Finally, we investigated whether such effects were observable on the level

187 of word-by-word processing during sentence reading, without averaging over trials, by quantifying

188 the improvement of MEG signal prediction in a comparative cross-validated model scheme.

189 2. Methods

190 2.1. Participants

191 Participants were 99 right-handed native Dutch speakers (age range 18-33 years; mean age = 22;

19250 males) from a subset of 102 participants who completed a reading paradigm in the open-access

193 MOUS dataset (Mother of all Unification Studies; Schoffelen et al., 2019). Three participants were

194 excluded from analyses, due to technical issues during data acquisition making them unsuitable

195 for the current analysis pipeline. All participants were right handed, had normal or corrected to

196 normal vision, and reported no history of neurological, developmental or language impairments.

197 All participants provided written informed consent and the study was approved by the local ethics

198 committee, and complied with the declaration of Helsinki.

\section{2.2. Sentence stimuli}

200 The total stimulus set consisted of 360 Dutch sentences (9-15 words in length), which are described

201 in detail in Schoffelen et al. (2019). Each participant read a selection of 240 sentences (2/3 of the

202 entire stimulus set), where $50 \%$ were presented as intact sentences and $50 \%$ were presented as

203 scrambled sentences. Specifically, three pairs of selections, referred to as scenario pairs, were

204 created, such that the stimuli that occurred as normal sentences in one scenario from a pair were 
205 presented in a scrambled fashion in the other scenario from that pair, and vice versa. Sentences

206 were scrambled so that no more than three words in a scrambled sentence made up a coherent

207 phrase. No participant read both the intact and scrambled version of a sentence. Consequently of

208 this design was that the collection of words that subjects read was exactly counterbalanced across

209 intact and scrambled sentence conditions, both across all participants and within the three sets of

210 scenario pairs.

\section{2.3. Lexical characteristics}

212 Lexical characteristics of frequency, index, surprisal, entropy and length (i.e. number of

213 characters) were obtained for each word in the sentence, to enter as predictors into regression

214 models. Lexical frequency was defined as the frequencies of words occurring in the NLCOW2012

215 corpus (Schäfer \& Bildhauer, 2012) and were $\log 10$ transformed. The NLCOW2012 database is

216 comprised of over 10 million Dutch sentences (71761868 words), and was also used to obtain

217 estimates of surprisal and entropy (see below). Index was defined as the ordinal position of the

218 word in the intact/scrambled sentence. Each word's surprisal value was acquired from a trained

219 tri-gram model, using WOPR (Van Den Bosch \& Berck, 2009), trained on the NLCOW2012

220 corpus. Surprisal was computed as the conditional probability of observing a word given the

221 previous two words in the sentence. Formally, it was computed as:

$$
\operatorname{surprisal}(w(t))=-\log P(w(t) \mid w(t-2), w(t-1))
$$

223 High surprisal values therefore signify low lexical predictability. Entropy was acquired from the

224 same trained tri-gram model. Entropy reflects the probability distribution of possible

225 continuations, given the constraints of the previous words. High entropy values signify a high

226 number of possible continuations, i.e. low predictability of the upcoming word. Formally, it is 227 defined as: 
$228 \quad \operatorname{entropy}(w(t))=-\sum_{w(t+1) \in W} P(w(t+1) \mid w(1), \ldots, w(t)) \log P(w(t+1) \mid w(1), \ldots, w(t))$

229 Using a trained tri-gram model here, the entropy at word $\mathrm{w}(\mathrm{t}+1)$ reflects the summation across all 230 possible endings given $\mathrm{w}(\mathrm{t})$ and $\mathrm{w}(\mathrm{t}-1)$.

231 All metrics were also computed for the first two words in a sentence. The statistical language 232 model allowed for estimates of sentence onset words (and also the second word in the sequence),

233 since sentences were prepended by special tokens, which allowed the first sentence word to be 234 treated as a valid trigram.

235 The distribution of the estimated surprisal values for both scrambled and intact sentences are 236 presented in Fig 1. Here it can be seen that model-based surprisal and entropy are higher for 237 scrambled than intact sentences. Although there were likely many trigrams in the current stimuli 238 that were not present in the corpus on which the language model was trained, particularly in the 239 scrambled sentence condition, N-gram based statistical language models account for this by 240 estimating the conditional probabilities using a technique called smoothing (or discounting), 241 returning non-zero probabilities for words, even if corresponding trigrams did not occur in the 242 training set. In such cases, the returned conditional probabilities will be more closely related to the 243 (unconditional) lexical frequency of the word. Fig 1 additionally highlights that measures of lexical 244 frequency and surprisal, and lexical frequency and length were highly correlated. This is 245 unsurprising, as both lexical frequency and surprisal were calculated from the frequency of 246 occurrences in a corpus, either of the word itself, or the word given the prior two words. Such high 247 correlations were not a concern for the current analysis, in which we used a model comparison 248 procedure to quantify the additional variance explained by a model including the independent 

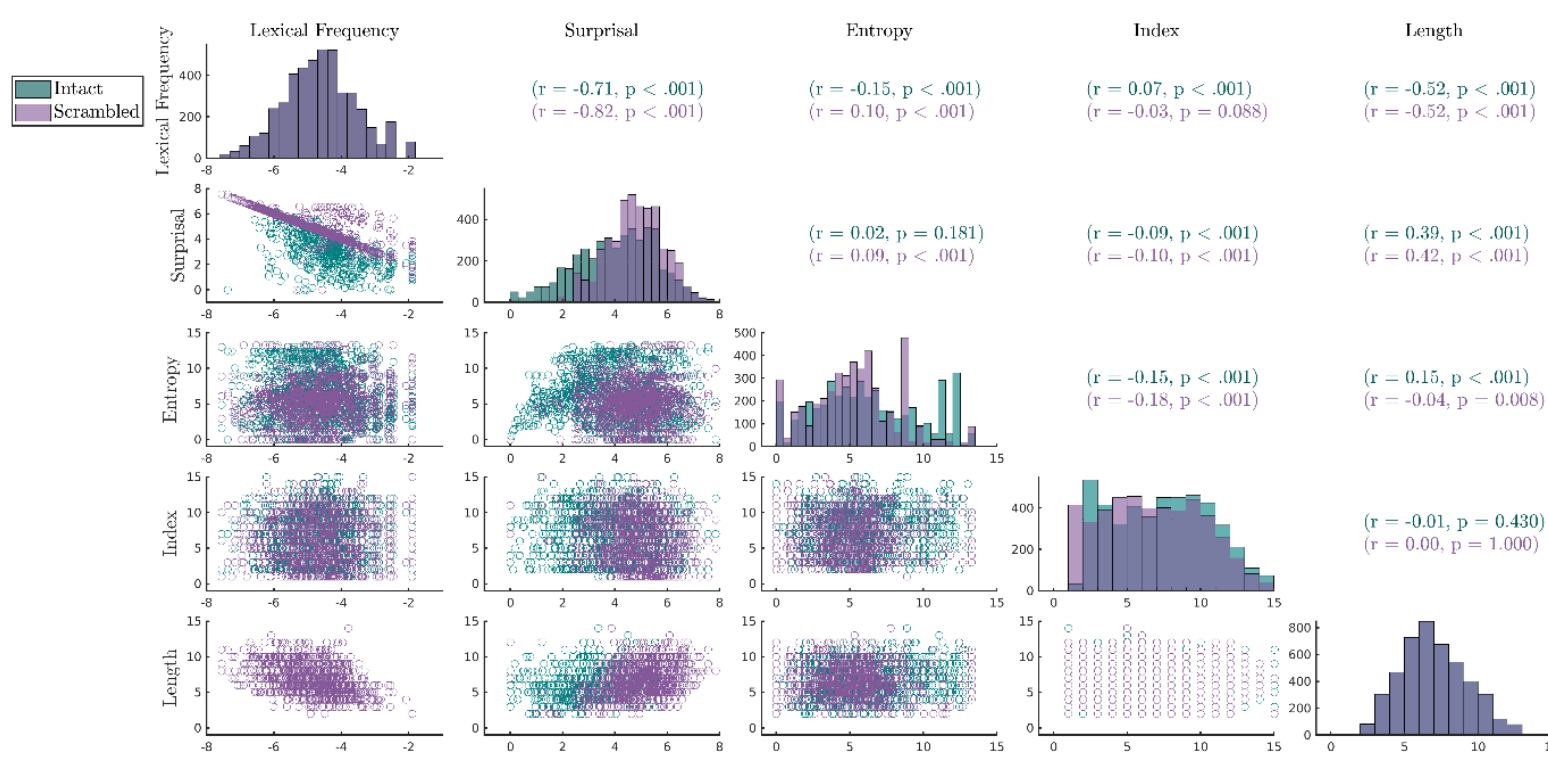

$-0.09, \mathrm{p}<.001)$
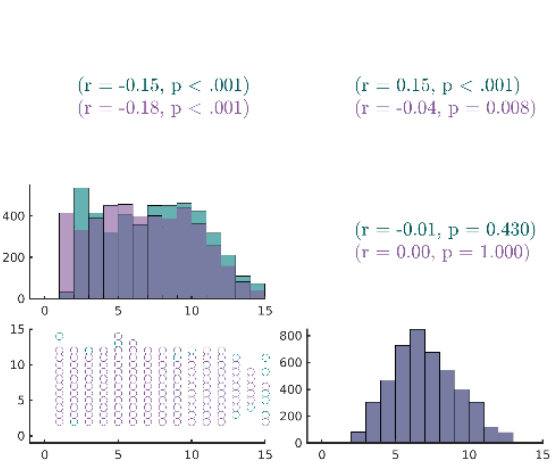

253

Figure 1. Correlation matrix for predictor variables lexical frequency (log10-transformed), surprisal ( $\log 10$-transformed), entropy, index and word length (respectively) for the content words. Scatterplots between corresponding pairs of predictors are presented in the lower off-diagonal.

256 Pearson's correlation coefficients and corresponding $\mathrm{p}$ values are presented on the upper offdiagonal. Histograms present the distribution of each predictor variable on the diagonal.

\subsection{Experimental procedure}

260 Sentence stimuli were presented in a random order in alternating intact and scrambled sentence

261 blocks. There were 48 blocks in total, each containing five intact sentences or five scrambled

262 sentences. The starting block condition (intact/scrambled) was randomised across participants. At

263 the beginning of each block the block type was presented on the screen for $1500 \mathrm{~ms}$. Trials

264 (intact/scrambled sentences) were separated with a 1200-2200 inter-trial interval, during which a

265 blank screen was presented followed by a fixation cross. Stimuli were presented word-by-word, 
266 with an inter-stimulus (word) interval of 300ms. To avoid the entrainment of neural oscillations to

267 a rhythmic onset of visual stimuli, and to better match the pace of the equivalent spoken stimuli

268 (Schoffelen et al., 2019), the presentation duration of each word was adjusted by the word duration

269 when spoken (visual presentation duration $=300-1400 \mathrm{~ms}$, mean $=351 \mathrm{~ms}$ ). The calculation of

270 single word durations has been described elsewhere (Lam, Schoffelen, Udden, Hulten, \& Hagoort,

271 2016; Schoffelen et al., 2019). To reiterate, for each intact/scrambled sentence, the duration of a

272 single word was a function of four factors: (i) the duration of the spoken version of the

273 intact/scrambled sentence in the matching auditory stimuli from Schoffelen et al. (2019)

274 (audiodur), (ii) the total number of words in the sentence (nwords), (iii) the number of letters per

275 word (nletters), and (iv) the total number of letters in the sentence (sumnletters). Single word

276 duration was computed as:

277 (nletters/sumnletters)*(audiodur+2000-150*nwords)

278 The minimum presentation duration for short words was limited to $300 \mathrm{~ms}$, regardless of the 279 outcome of the above formula. As the presentation rate of stimuli was partially determined by the 280 refresh rate of the projector $(60 \mathrm{~Hz})$, the actual presentation duration of words increased by $0-33 \mathrm{~ms}$

281 from the value provided by the above formula.

282 Participants were instructed to read the sentences. On 20\% of trials participants answered a yes/no 283 comprehension question to ensure they were engaged in the task. The positions of the 284 comprehension questions relative to the stimuli were random. In intact sentence blocks, $50 \%$ of 285 questions asked about the content of the sentence (e.g. "Did grandma give a cookie to the girl?").

286 Questions in the scrambled sentence blocks, and the remaining $50 \%$ of questions in the intact 287 sentence blocks, asked about the presence of a content word (e.g. "Was the word grandma 
mentioned?"). Participants responded to the questions by pressing a button with their left

289 index/middle finger to answer yes/no, respectively.

290 Stimuli were presented with Presentation software (Version 16.0, Neurobehavioral Systems, Inc)

291 and back-projected with an LCD projector at a refresh rate of $60 \mathrm{~Hz}$. Words were presented in the

292 centre of the screen in a black mono-spaced font (visual angle of 4 degrees) on a grey background.

293 Before beginning the main experiment, participants completed practice trials to familiarise

294 themselves with the procedure.

\section{2.5. MEG acquisition}

296 Participants were seated in a magnetically shielded room, while MEG was recorded with a 275

297 axial gradiometer CTF system, at a sampling rate of $1200 \mathrm{~Hz}$ and with a $300 \mathrm{~Hz}$ analog low pass

298 filter. Prior to the recording, the participant's head shape was digitised with a Polhemus 3D-Space

299 Fast-track digitiser. Digitised head shapes and fiducial points were later used to coregister subject-

300 specific anatomical MRIs with the MEG sensor space. The position of the participants' head

301 (relative to the MEG sensors) was monitored online throughout the recording via three head-

302 localiser coils, placed on the nasion and left and right pre-auricular points.

\section{2.6. MRI acquisition}

304 MRIs were recorded with a Siemens Trio 3T MRI scanner with a 32-channel head coil. A T1305 weighted magnetisation-prepared rapid acquisition gradient echo pulse sequence was used to 306 obtain structural MRIs (volume TR $=2300 \mathrm{~ms} ; \mathrm{TE}=3.03 \mathrm{~ms} ; 8^{\circ}$ flip angle; 1 slab; slice matrix size $307=256 \times 256 ;$ slice thickness $=1 \mathrm{~mm} ;$ field of view $=256 \mathrm{~mm} ;$ isotropic voxel size $=1.0 \times 1.0 \times$ 308 1.0mm). A vitamin E capsule was placed behind the right ear as a fiducial marker to visually 309 identify left/right. 


\subsection{Data analysis}

311 Pre-processing

312 Data were band pass filtered between $0.5-20 \mathrm{~Hz}$ and epoched time-locked to sentence onset.

313 Segments of data that contained eye blinks, squid jumps and muscle artifacts were replaced with

314 "Not a Number" (NaN) in order to preserve the original sentence onset related timing information.

315 Data were downsampled to $120 \mathrm{~Hz}$.

\section{Source Reconstruction}

317 Single shell head models describing the inside of the skull were constructed from individual MRIs, 318 which were used to create forward models according to (Nolte, 2003). Single trial covariance 319 matrices were computed between sensor pairs. Sources were reconstructed using linearly 320 constrained minimum variance (LCMV; Van Veen, van Drongelen, Yuchtman, \& Suzuki, 1997) 321 beamforming to obtain time courses of source activity at 8196 dipole locations. Data were 322 parcellated using an anatomical atlas-based parcellation, consisting of 382 parcels (Schoffelen et 323 al., 2017). For each parcel, principal component analysis was performed on the dipole time series 324 belonging to a given parcel, and the top five components that explained the most variance in the 325 parcel-specific signal were selected for further analysis.

\section{Spatiotemporal Alignment}

327 To boost the stimulus specific signal, and reduce intersubject variability, data were 328 spatiotemporally aligned across subjects using multiset canonical correlation analysis (MCCA;

329 Arana et al., 2020; de Cheveigné et al., 2019). MCCA was used to find linear combinations of the 33065 parcel time courses (canonical components) that maximised the correlation between all subject 
331 pairs, while they were presented with exactly the same words, thereby increasing the similarities

332 between the participants' signals in response to those words.

333 MCCA is a generalization of canonical correlation analysis (CCA), and aims to find linear

334 combinations for multivariate observations in order to maximize the correlation between the

335 combined time series. Here, each member of the set of multivariate observations consisted of a

336 representation of a parcel-specific signal for a given subject. Linear combinations of these

337 observations were estimated, which resulted in a single canonical component per subject such that

338 the correlation across subjects was maximised. The linear weights were estimated with a

339 generalized eigenvalue decomposition using two covariance matrices, consisting of the full

340 covariance matrix of all subjects' multivariate observations, and of a block-diagonal covariance

341 matrix, containing only the within subject covariances of the multivariate observations. As

342 mentioned, our aim was to boost the stimulus-specific brain signals, specifically accounting for

343 some spatial and temporal variability across subjects. Hence, for each subject the input to MCCA

344 decomposition consisted of a set of time-shifted time series, where the parcel's 5 dominant

345 principal components were shifted in time from -50-50ms in steps of single samples, resulting in

34665 time series per word per parcel and subject (i.e. 5 principal components $\times 13$ time shifts).

347 MCCA was performed separately for each pair of scenarios, which were fully matched in terms of

348 the stimulus material that was used to derive the sentences and the word-lists (i.e. the subjects read

349 exactly the same overall collection of individual words), based on combining data from sets of $32-$

35034 subjects. Next, the time series of the scrambled sentence trials were unscrambled such that the

351 word order and onset times exactly matched the corresponding intact sentence's word order and

352 onset times. This resulted in 240 trials that were exactly matched across time in terms of the

353 individual words presented. These trials were entered into a five-fold cross validated MCCA 
354 procedure (Arana et al., 2020). To this end, we partitioned the data into 5 test folds of 48 trials

355 each, and for each of the folds used the 192 remaining trials as a training set to estimate the MCCA

356 weights. These weights were subsequently applied to the test fold data to obtain the subject-

357 specific canonical components. The cross validation was applied in order to avoid overfitting. To

358 summarise, MCCA was used to find linear combinations of the 65 parcel time courses (canonical

359 components) that maximised the correlation between all subject pairs, while they were presented

360 with exactly the same words, thereby increasing the similarities between the participants' signals

361 in response to those words.

362 Encoding Models

363 Next, we fitted encoding models to the data, using five-fold cross-validated ridge regression. To

364 this end, the subject-specific canonical components were re-epoched time-locked to word onset,

365 selecting only content words (nouns, adjectives, and verbs). The content words made up 55\% of

366 all the words in the stimulus set, which resulted in an average of 763 (range: 755-774) words per

367 scenario and main condition (intact versus scrambled sentences). The absolute number of analysed

368 words per sentence varied as a function of sentence length. For the re-epoched data, subject-

369 specific encoding models were estimated for each time point and parcel-of-interest, separately for

370 intact and scrambled sentence words. A ridge regression model is similar to a multiple regression

371 model with a regularised design covariance matrix. The optimal regularisation parameter was

372 estimated using nested cross-validation, and selected from a range of lambda values $(0.002,0.005$,

$3730.010,0.020,0.050,0.100,0.200,0.500,1.000,2.000$ and 5.000) for each model. The

374 regularisation parameter applies a penalty to the model to avoid overfitting on the training data. A

375 lambda value of 0 would result in no regularisation being applied, whereas selecting a lambda 
376 value that is too high would result in under-fitting the model. The model derived from a "training"

377 portion of the data was evaluated on its performance to predict a portion of unseen "test" data.

378 In order to separate the unique variance explained by each variable of interest from that explained

379 by all other variables, we applied a model comparison scheme. The model comparison procedure

380 quantified the extent to which a model including a predictor of interest explained variance in the

381 MEG signal, above and beyond a reduced model that did not include the given predictor. To this

382 end we computed the coefficient of determination:

$$
R^{2}=1-\frac{\sum\left(y-\hat{y}_{\text {full model }}\right)^{2}}{\sum\left(y-\hat{y}_{\text {reduced model }}\right)^{2}}
$$

384 Where the numerator and denominator in the right side of the equation were computed as the sum385 of-squares of the difference between the data and the modelled test data, for the full and reduced 386 models, respectively.

387 To test the contribution of individual predictors we used a full model that included, beyond a 388 constant and word length, the following predictors of interest: lexical frequency (log transformed), 389 surprisal, entropy and index. To test the interaction between lexical frequency and context - as 390 quantified with index (similar to Alday et al., 2017; Payne et al., 2015; Van Petten \& Kutas, 1990) 391 - we used a full model that included only, beyond a constant, the individual predictors of lexical 392 frequency (log transformed), index, length, and the interaction term, which was computed as an 393 element wise product: lexical frequency $(\log 10$ transformed $) \times$ index. Similarly, we tested the 394 interaction between lexical frequency and surprsial, and lexical frequency and entropy, where the 395 full model included, beyond the constant, the individual predictors of lexical frequency (log 396 transformed), surprisal (log transformed)/entropy, length, and the interaction term (lexical 397 frequency $\times$ surprisal $(\log$ transformed)/lexical frequency $\times$ entropy). Epochs (content words) were 
398 divided into five equal folds to avoid overfitting, and to allow for the generalisation across items.

399 For each fold of the cross-validation procedure, the model was estimated using data from the four

400 other folds, and tested on the remaining data.

401 In order to be able to statistically compare the models for the individual intact and scrambled

402 sentence conditions, that is to obtain an estimate of a possible bias in the coefficient of

403 determination under the null hypothesis, we used a permutation approach, as follows: For each

404 model, the design matrix was randomly permuted 50 times and, for each permutation, an additional

405 model was trained and tested with the permuted variables, thereby removing any true association

406 between the predictors and the data.

407 Statistical Analysis

408 We statistically evaluated the individual predictors in a selection of regions-of-interest (ROI), 409 consisting of 184 parcels (92 left hemisphere parcels with their right hemisphere counterparts).

410 This selection consisted of cortical regions that have consistently been described to be a part of a 411 language network (Catani et al., 2007; Friederici, 2009; Glasser \& Rilling, 2008; Schoffelen et al., 412 2017) or to be involved in the processing of semantic relationships (Bunge, Helskog, \& 413 Wendelken, 2009; Frankland \& Greene, 2020; Knowlton, Morrison, Hummel, \& Holyoak, 2012;

414 Ramnani \& Owen, 2004). We further investigated the interaction between lexical frequency and 415 index based on the resulting map including only the 33 parcels that significantly encoded index or 416 lexical frequency. The interaction between lexical frequency and surprisal, and lexical frequency 417 and entropy were investigate in the same 33 parcels, facilitating comparison across results.

418 We used non-parametric permutation statistics, using the dependent samples T-statistic across 419 subjects as a test statistic. We evaluated the individual coefficients of determination against the 
420 corresponding average of their 50 random permutation counterparts (see Encoding Models section

421 2.7), using an alpha-level of 0.05 for inference. The intact and scrambled sentence conditions were

422 compared with each other using a two-sided test (which involves evaluating the test statistic

423 against two randomisation distributions, using an alpha level of 0.025 for each of these

424 randomisation distributions) for inference. For all comparisons, multiple comparisons (across time

425 and space) were accounted for by using a max-statistic distribution from 5000 permutations.

426 Note that we compared intact and sentence conditions only on the difference in the interaction

427 between lexical frequency and index, and not in the interaction between lexical frequency and

428 surprisal, nor lexical frequency and entropy. Index is well-controlled across intact/scrambled

429 sentence conditions, in that it is well matched across both intact and scrambled sentences, and does

430 not correlate with lexical frequency. In contrast, the distribution of surprisal and entropy both differ

431 across intact and scrambled sentences, with higher surprisal and entropy values in scrambled

432 compared to intact sentences (see Fig 1). Any observed difference between intact and scrambled

433 sentences in the variance explained by the interaction between lexical frequency and

434 surprisal/entropy could, therefore, be down to their different distributions of surprisal/entropy 435 values.

\section{3. Results}

437 All participants achieved over 60\% accuracy on the comprehension questions (mean $=81.19 \%$; sd $438=6.61 \%$ ), confirming they were attending to the stimuli. No further analysis was conducted on the 439 comprehension questions. 


\subsection{Spatiotemporal Alignment}

441 Fig 2 shows the effect of the alignment procedure, presenting the time-resolved intersubject

442 correlation (Fisher Z-transformed correlation coefficient) after spatiotemporal alignment (solid

443 green line), spatial alignment (dashed green line), temporal alignment (dotted red line) and no

444 alignment (dashed purple line), for two example parcels (sub-regions of BA22 and BA44). Fig 2

445 illustrates that spatiotemporal alignment increased the intersubject correlation, more so than

446 temporal alignment alone or spatial alignment alone. The intersubject correlation peaked at around

$447400 \mathrm{~ms}(300-500 \mathrm{~ms})$, a time period in which electrophysiological brain signal is typically found to

448 be influenced by the semantic characteristics of a word (N400/M400; Kutas \& Federmeier, 2011).

449 Spatiotemporal alignment thereby seems to have boosted the stimulus specific signal in the data.

A

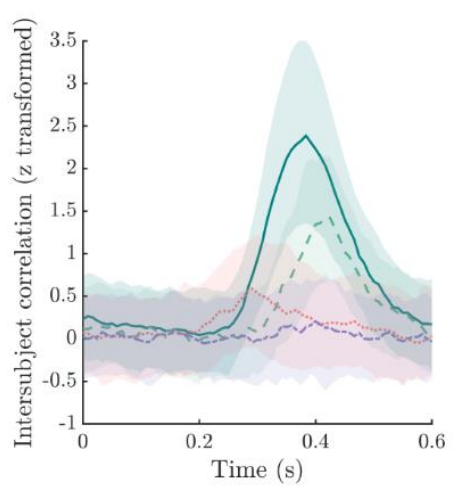

B

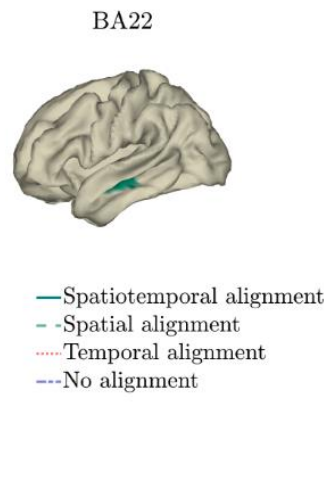

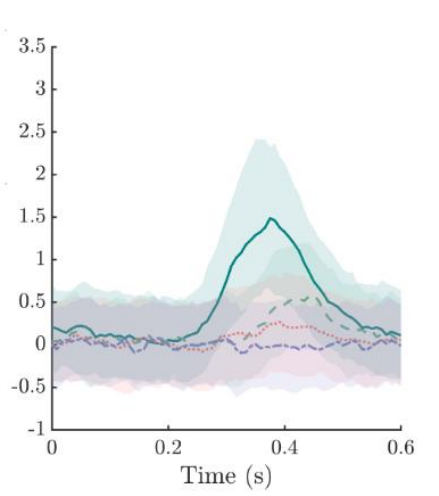

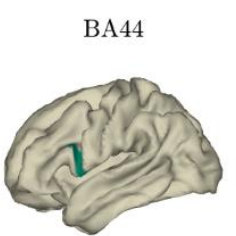

-Spatiotemporal alignment

- -Spatial alignment

-... Temporal alignment

---No alignment

Figure 2. MCCA boosts intersubject consistency of single word responses. Time courses of Ztransformed intersubject correlations after spatiotemporal alignment (solid green line), spatial alignment (dashed green line), temporal alignment (dotted red line) and no alignment (dashed purple line) in middle temporal gyrus (parcel in Brodmann Area (BA) 22; panel A) and inferior frontal gyrus (parcel in BA44; panel B). Shaded ribbons represent the interquartile range.

\subsection{Encoding Models}

For each measure of interest, our model comparison scheme quantified the extent that each regressor explained word-specific variance in the MEG signal, beyond the variance explained by all other regressors (see Methods section 2.7). Similarly, we quantified the variance explained by 
461 the index $\times$ lexical frequency interaction, surprisal $\times$ lexical frequency interaction and entropy $\times$

462 lexical frequency interaction, beyond that explained by the main effects of lexical frequency and

463 index/surprisal/entropy (respectively). The model comparisons were statistically evaluated

464 separately for the intact (Figs 3-9 panel A) and scrambled (Figs 3-7 panel B) sentences against a

465 permutation derived baseline, as well as compared against each other (Figs 3-7 panel C).

\section{Lexical Frequency}

467 Lexical frequency significantly predicted MEG signal in both intact and scrambled sentences

468 throughout the $0-600 \mathrm{~ms}$ analysis window (relative to word onset), spatially spreading from

469 bilateral occipital and inferior temporal cortex to left posterior and middle temporal cortex at time

470 points preceding $250 \mathrm{~ms}$, to left frontal and left anterior temporal cortex from $250 \mathrm{~ms}$ onwards. In

471 both intact and scrambled sentences, the effect of lexical frequency peaked at around 400ms in left

472 temporal and frontal cortex (Fig 3 panels A and B). In the left superior temporal gyrus (STG) and

473 middle temporal gyrus (MTG) this effect started earlier in intact compared to scrambled sentences,

474 from $183 \mathrm{~ms}$, compared to $267 \mathrm{~ms}$ in scrambled sentences.

475 Despite the seemingly stronger effect in scrambled compared to intact sentences - apparent in the 476 time courses in Fig 3 panel D - in a direct comparison of the coefficient of determination for lexical

477 frequency across conditions (presented in Fig 3 panel C), only a very small spatiotemporal effect 478 survived the multiple comparisons correction scheme. Specifically, significantly more variance 479 was explained in intact compared to scrambled sentences at a single time point, at $267 \mathrm{~ms}$, in a 480 single right hemisphere frontal parcel (BA46). There were no other significant differences between 481 intact and scrambled sentences in the variance explained by lexical frequency (corrected $p>.05$ ). 


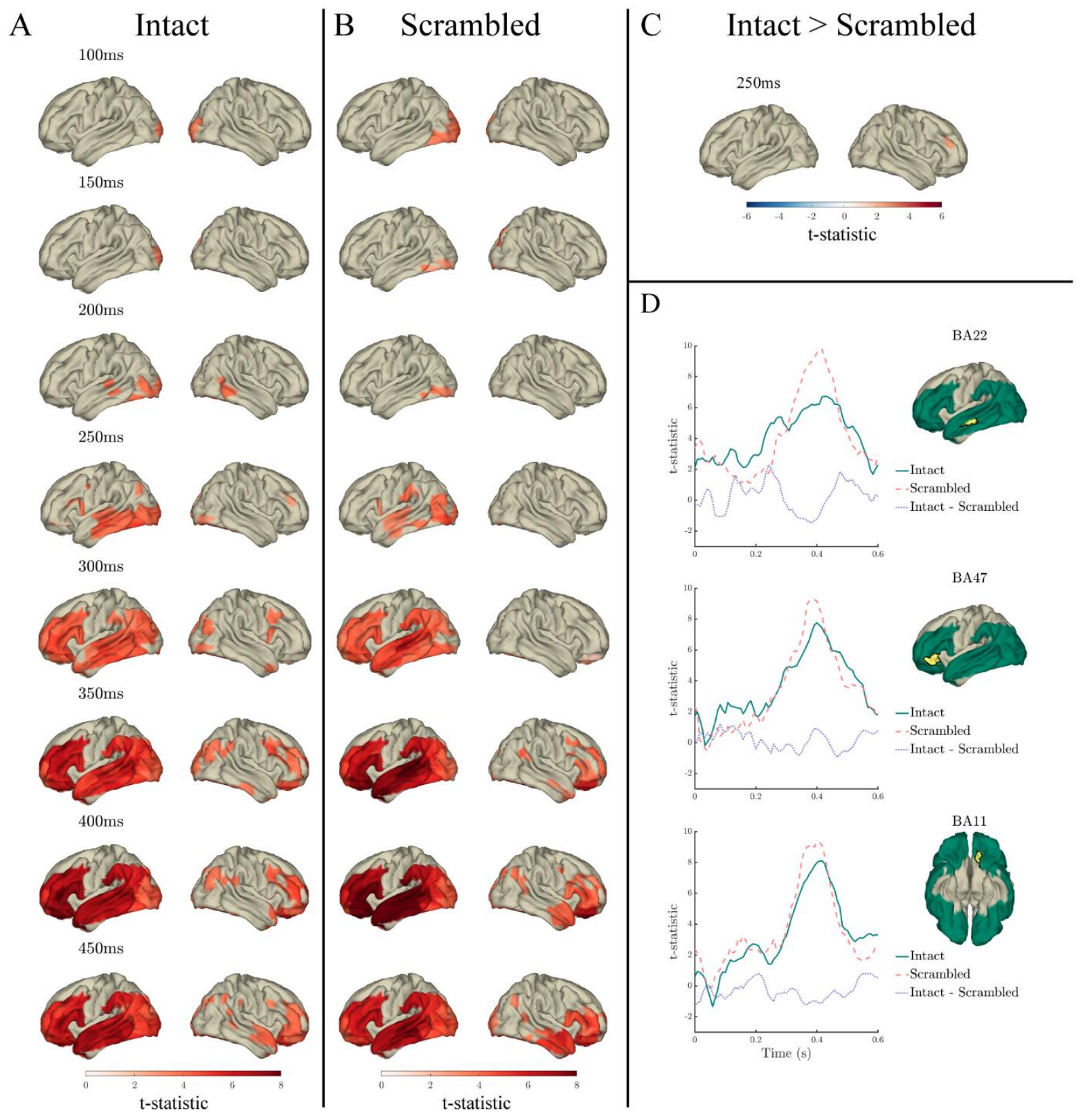

484 Figure 3. Effects of lexical frequency in the response to content words: Surface plots of T-statistics 485 (averaged over 50ms time windows centred at the indicated latencies, for visualisation) quantifying 486 the difference in variance explained by lexical frequency (log10 transformed), beyond that 487 explained by index, surprisal, entropy and length, in intact sentence compared to random permutation models (panel A; $p<.05$ one-sided, corrected), scrambled sentence compared to random permutation models (panel $\mathrm{B} ; p<.05$ one-sided, corrected), and intact compared to scrambled sentence models (panel $\mathrm{C} ; p<.05$ two-sided, corrected). Parcels for which no time point was significant during the $50 \mathrm{~ms}$ time bin are masked. Panel D: Time courses of T-statistics for intact (solid green line) and scrambled (dashed red line) sentence models compared to random permutation models, and intact compared to scrambled sentence models (dotted purple line) for subparcels of BA22, BA47 and BA11 (highlighted in yellow on adjacent surface plots). ROIs entered into statistical analyses are illustrated as green shaded area on surface plots. 
497 Index significantly predicted the MEG signal in both intact and scrambled sentences throughout 498 the $0-600 \mathrm{~ms}$ analysis window. In intact sentences the effect spread from bilateral occipital cortex 499 throughout right posterior and inferior temporal cortex and left temporal and frontal cortex, and 500 peaked at around 350ms in left anterior temporal and inferior frontal cortex (Fig 4 panel A). In 501 contrast to intact sentences, in scrambled sentences the effect was predominantly constrained to 502 bilateral occipital and inferior temporal cortex, peaked at around $300 \mathrm{~ms}$ in left posterior and 503 inferior temporal cortex (Fig 4 panel B), and after 492ms only two single time points were 504 significant (542ms and 600ms).

505 Significantly more variance in the MEG signal was predicted by index in intact compared to 506 scrambled sentences from 275-417ms in anterior temporal (BA21/22/38), 283-375ms in inferior 507 frontal (BA44/46/47), and 258-400ms in orbitofrontal and prefrontal cortex (PFC; BA10/11), as 508 is evident in Fig 4 panel C. 


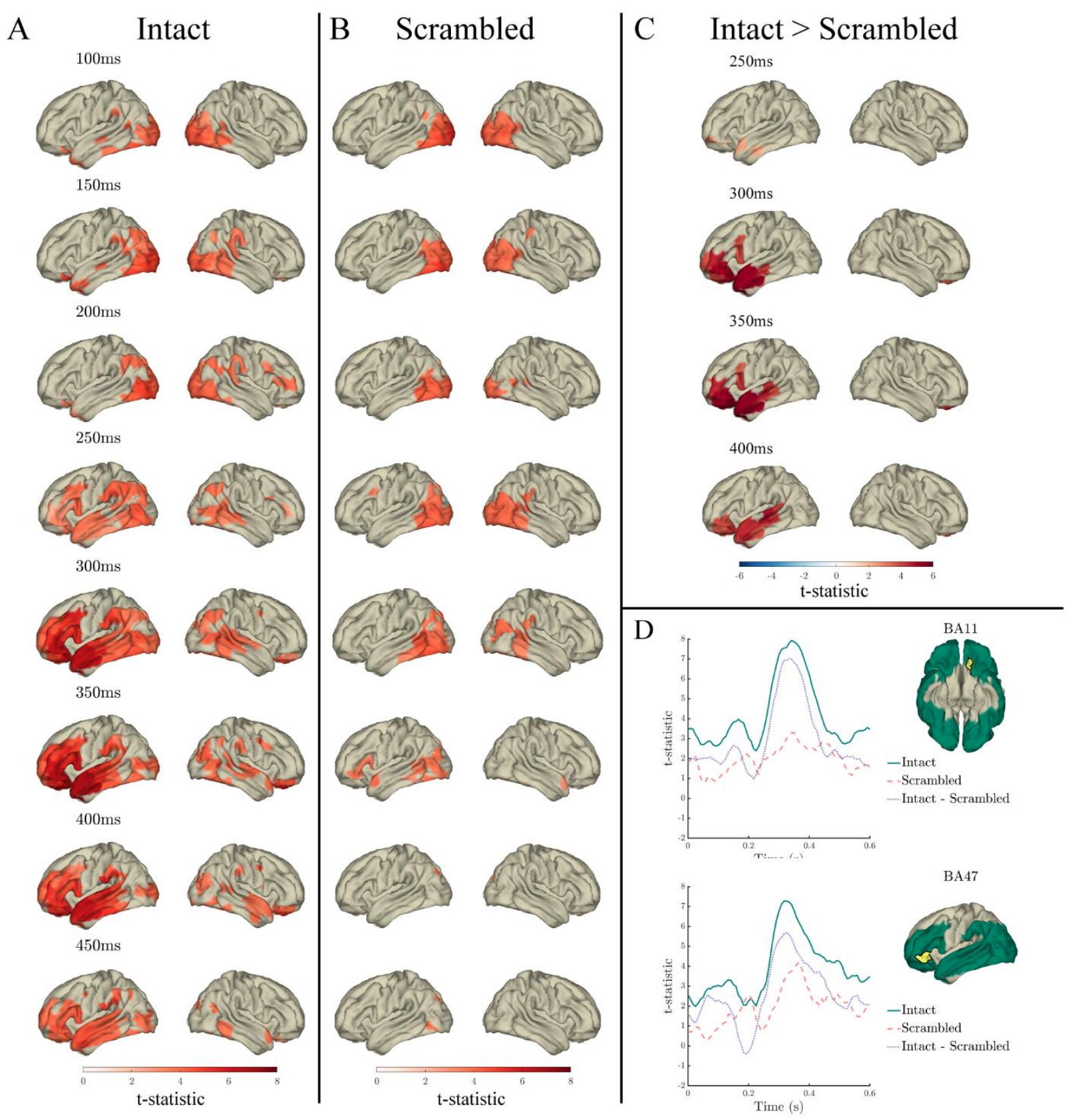

510 Figure 4. Effects of index in the response to content words: Surface plots of T-statistics (averaged 511 over $50 \mathrm{~ms}$ time windows centred at the indicated latencies, for visualisation) quantifying the 512 difference in variance explained by index, beyond that explained by lexical frequency $(\log 10$ 513 transformed), surprisal, entropy and length, in intact sentence compared to random permutation 514 models (panel A; $p<.05$ one-sided, corrected), scrambled sentence compared to random 515 permutation models (panel $\mathrm{B} ; p<.05$ one-sided, corrected), and intact compared to scrambled 516 sentence models (panel $\mathrm{C} ; p<.05$ two-sided, corrected). Parcels for which no time point was 517 significant during the 50ms time bin are masked. Panel D: Time courses of T-statistics for intact 518 (solid green line) and scrambled (dashed red line) sentence models compared to random 519 permutation models, and intact compared to scrambled sentence models (dotted purple line) for 520 subparcels of BA11 and BA47 (highlighted in yellow on adjacent surface plots). ROIs entered into 521 statistical analyses are illustrated as green shaded area on surface plots. 


\section{Surprisal}

524 In both intact and scrambled sentences, surprisal significantly predicted the MEG signal

525 throughout most of the analysis window, peaking at 400ms in temporal and frontal cortex, and

526 predicting additional right hemisphere variance in orbitofrontal and anterior temporal cortex (see

527 Fig 5 panels A and B). In intact sentences, the effect spread from STG and the angular gyrus from

528 0-100ms, throughout temporal and frontal cortex from 208-600ms (Fig 5 panel A). In scrambled

529 sentences, the effect spread from left (later bilateral) occipital and inferior temporal cortex

530 throughout primarily the left temporal and frontal cortex (Fig 5 panel B). However, the effect of

531 surprisal in scrambled sentences was most robust from 200ms onwards. Preceding 200ms, only

532 several individual time points were significant after multiple comparisons correction.

533 Significantly more variance in the MEG signal was predicted by surprisal in intact compared to 534 scrambled sentences $50-58 \mathrm{~ms}$ and $458-475 \mathrm{~ms}$ relative to word onset in left MTG (BA22), and 535 392-442ms relative to word onset in bilateral orbitofrontal cortex (BA11), which is presented in

536 Fig 5 panel C. The time courses in Fig 5 panel D illustrate that the significant difference in BA22 537 results from a more sustained response in intact compared to scrambled sentences, whereas BA11 538 results from a greater peak in intact compared to scrambled sentences. 


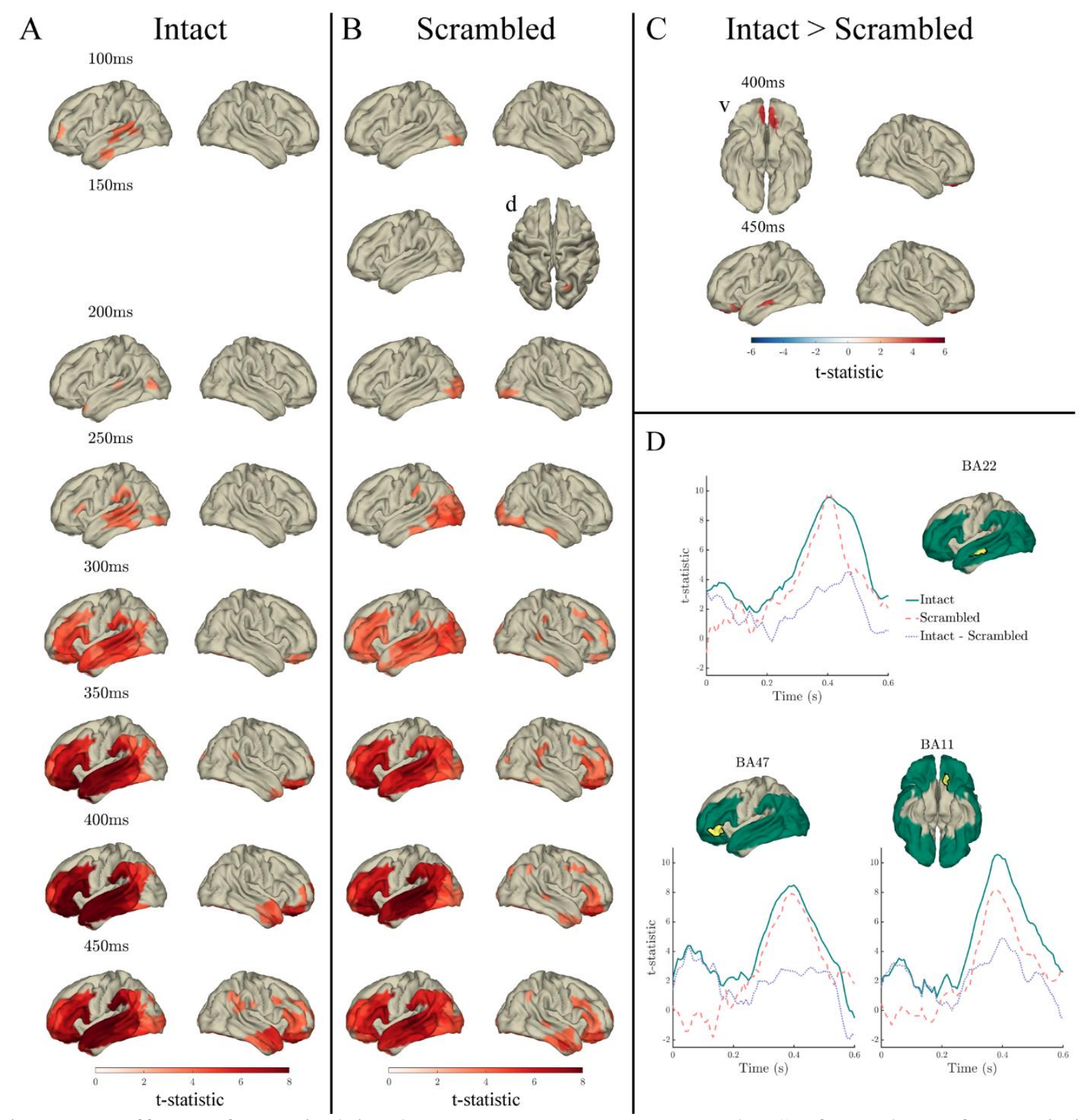

Figure 5. Effects of surprisal in the response to content words: Surface plots of T-statistics (averaged over 50ms time windows centred at the indicated latencies, for visualisation) quantifying 542 the difference in variance explained by surprisal, beyond that explained by lexical frequency 543 ( $\log 10$ transformed), index, entropy and length, in intact sentence compared to random 544 permutation models (panel A; $p<.05$ one-sided, corrected), scrambled sentence compared to random permutation models (panel $\mathrm{B} ; p<.05$ one-sided, corrected), and intact compared to scrambled sentence models (panel $\mathrm{C} ; p<.05$ two-sided, corrected). Parcels for which no time point was significant during the $50 \mathrm{~ms}$ time bin are masked. Ventral and dorsal views are indicated with adjacent "v" and "d" labels, respectively. Panel D: Time courses of T-statistics for intact (solid green line) and scrambled (dashed red line) sentence models compared to random permutation models, and intact compared to scrambled sentence models (dotted purple line) for subparcels of BA22, BA47 and BA11 (highlighted in yellow on adjacent surface plots). ROIs entered into statistical analyses are illustrated as green shaded area on surface plots. 
554 Entropy

555 Entropy significantly predicted the MEG signal in both intact and scrambled sentences, however

556 to a lesser extent than the aforementioned predictors. In intact sentences (see Fig 6 panel A),

557 entropy predicted variance in bilateral occipital, left inferior temporal and frontal parcels, and in

558 the posterior MTG, from 0-242ms, 292-367ms, and at individual time points of $433 \mathrm{~ms}$ and $525 \mathrm{~ms}$

559 (relative to word onset). In scrambled sentences (see Fig 6 panel B), entropy significantly predicted

560 variance in bilateral occipital and inferior temporal cortex from $0-83 \mathrm{~ms}$. Significantly more

561 variance was explained by entropy in intact compared to scrambled sentences in a single left

562 inferior temporal parcel (BA37) 450-458ms after word onset (see Fig 6 panel C). 


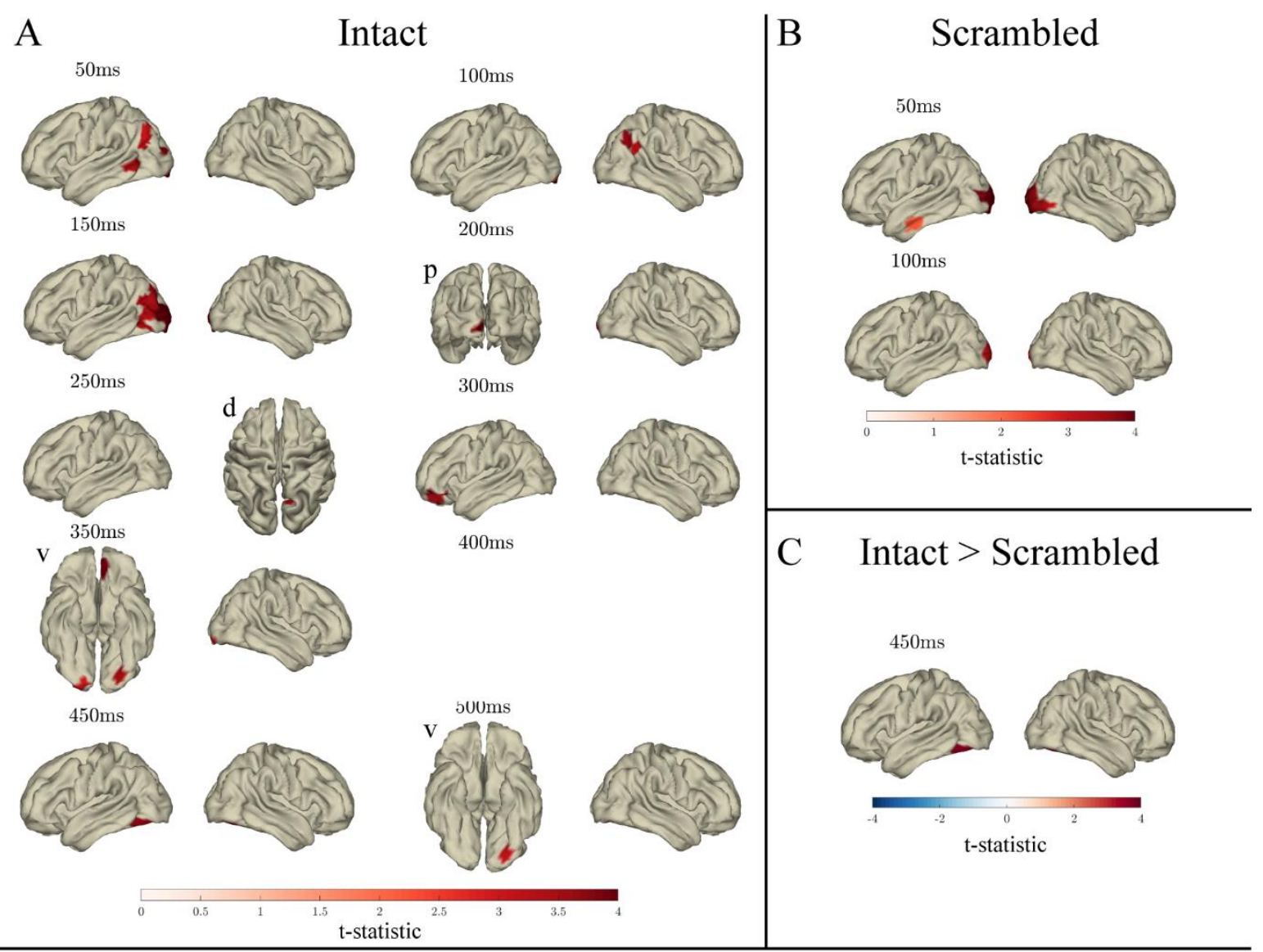

$\mathrm{D}$
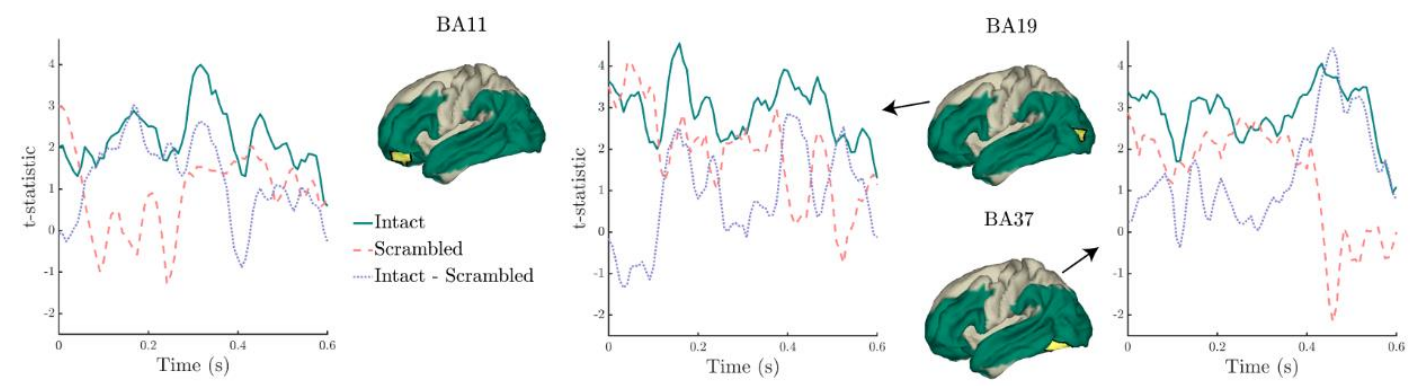

Figure 6. Effects of entropy in the response to content words: Surface plots of T-statistics (averaged over $50 \mathrm{~ms}$ time windows centred at the indicated latencies, for visualisation) quantifying the difference in variance explained by entropy, beyond that explained by lexical frequency $(\log 10$ transformed), index, surprisal and length, in intact sentence compared to random permutation models (panel A; $p<.05$ one-sided, corrected), scrambled sentence compared to random permutation models (panel $\mathrm{B} ; p<.05$ one-sided, corrected), and intact compared to scrambled sentence models (panel $\mathrm{C} ; p<.05$ two-sided, corrected). Parcels for which no time point was significant during the $50 \mathrm{~ms}$ time bin are masked. Ventral, dorsal, and posterior views are indicated with adjacent " $v$ ", "d" and "p" labels, respectively. Panel D: Time courses of T-statistics for intact (solid green line) and scrambled (dashed red line) sentence models compared to random permutation models, and intact compared to scrambled sentence models (dotted purple line) for subparcels of BA11, BA19 and BA37 (highlighted in yellow on adjacent surface plots). ROIs entered into statistical analyses are illustrated as green shaded area on surface plots. 
579 The above findings show how our model comparison approach identified brain activity patterns

580 that were aligned with word-by-word fluctuations of various quantities that relate to lexical

581 predictability. Considering that the interaction between lexical frequency and context (often

582 quantified with word position in the sentence) has been consistently reported in previous

583 electrophysiological studies (Alday et al., 2017; Dambacher et al., 2006; Payne et al., 2015; Sereno

584 et al., 2019; Van Petten \& Kutas, 1990), we conducted an analysis of this interaction in our data -

585 in parcels that showed conditional differences in effects of either lexical frequency or index (see

586 Figs 3-4) - specifically focussing on the spatial and temporal dynamics of this effect.

587 The interaction between lexical frequency and increased word position in the sentence is thought

588 to occur through the increasingly constraining context facilitating predictability as the sentence

589 progresses (Dambacher et al., 2006; Payne et al., 2015; Van Petten \& Kutas, 1990). Indeed, as

590 outlined in the Introduction, effects of lexical frequency and word predictability have been found

591 to interact (Dambacher et al., 2012; Dambacher et al., 2006; Kretzschmar et al., 2015; Sereno et

592 al., 2003; Sereno et al., 2019). Hence, in addition to investigating the interaction between lexical

593 frequency and index, we conducted an analysis of the interaction between lexical frequency and

594 measures of local predictability, surprisal and entropy. Given that there were only sparse

595 differences between intact and scrambled sentences in the effects of surprisal and entropy,

596 suggesting that surprisal and entropy quantify similar processing mechanisms regardless of the

597 level of sentential context, these interactions were investigated in sentences only, and in the same

598 parcels in which the lexical frequency $\times$ index interaction was investigated, in order to remain

599 consistent across analyses. 
600 Only effects of the interaction between lexical frequency and surprisal survived the stringent 601 multiple comparisons correction ( $p<.05$ corrected), and not the interactions with index and entropy.

602 Fig 8 presents the spatiotemporal distributions, along with example time courses, of T-statistics

603 for parcels and time points that were significant while correcting for multiple comparisons $(p<.05$

604 corrected), whereas Figs 7 and 9 present those that were significant without correcting for multiple 605 comparisons (uncorrected $p<.05$ ).

606 Lexical frequency $\times$ Index

607 Beyond the variance explained by the main effects of index, lexical frequency, and length the index $608 \times$ lexical frequency interaction explained additional variance in intact sentences from $150 \mathrm{~ms}$ after 609 word onset in frontal parcels (BA10/BA11/BA44/BA47), spreading to MTG and posterior STG 610 (BA22/BA38) from 342ms onwards, where effects peaked at around 400ms (see Fig 7 panel A; 611 uncorrected $p<.05)$.

612 In scrambled sentences, the index $\times$ lexical frequency interaction explained additional variance in 613 several time windows throughout the $0-600 \mathrm{~ms}$ analysis window, predominantly from $300 \mathrm{~ms}$ 614 onwards, but also at earlier time points. The effect spread from frontal (BA10/BA11) to temporal 615 (BA22/BA38) and inferior frontal (BA44/BA46) parcels, peaking at around 450ms in frontal 616 parcels (see Fig 7 panel B; uncorrected $p<.05$ ).

617 On inspection of Fig 7 panel $\mathrm{C}$, the comparison of the coefficient of determination for the 618 interaction in intact and scrambled sentence models revealed an interesting spatiotemporal pattern 619 of results. During an earlier time window (100-300ms), more variance was explained by the index $620 \times$ lexical frequency interaction in intact compared to scrambled sentences in frontal parcels 621 (BA10/BA11; warm colours Fig 7 panel C), yet more variance was explained by the index $\times$ lexical 622 frequency interaction in scrambled compared to intact sentences in temporal parcels 
623 (BA21/BA22/BA38; cool colours Fig 7 panel C). However, in a later time window (350-500ms)

624 a reverse pattern was observed, where more variance was explained by the interaction in scrambled

625 compared to intact sentences in frontal parcels, and more variance was explained in intact

626 compared to scrambled sentences in temporal parcels. This pattern is also evident in the time

627 courses of T-statistics presented in Fig 7 panel D.

628 


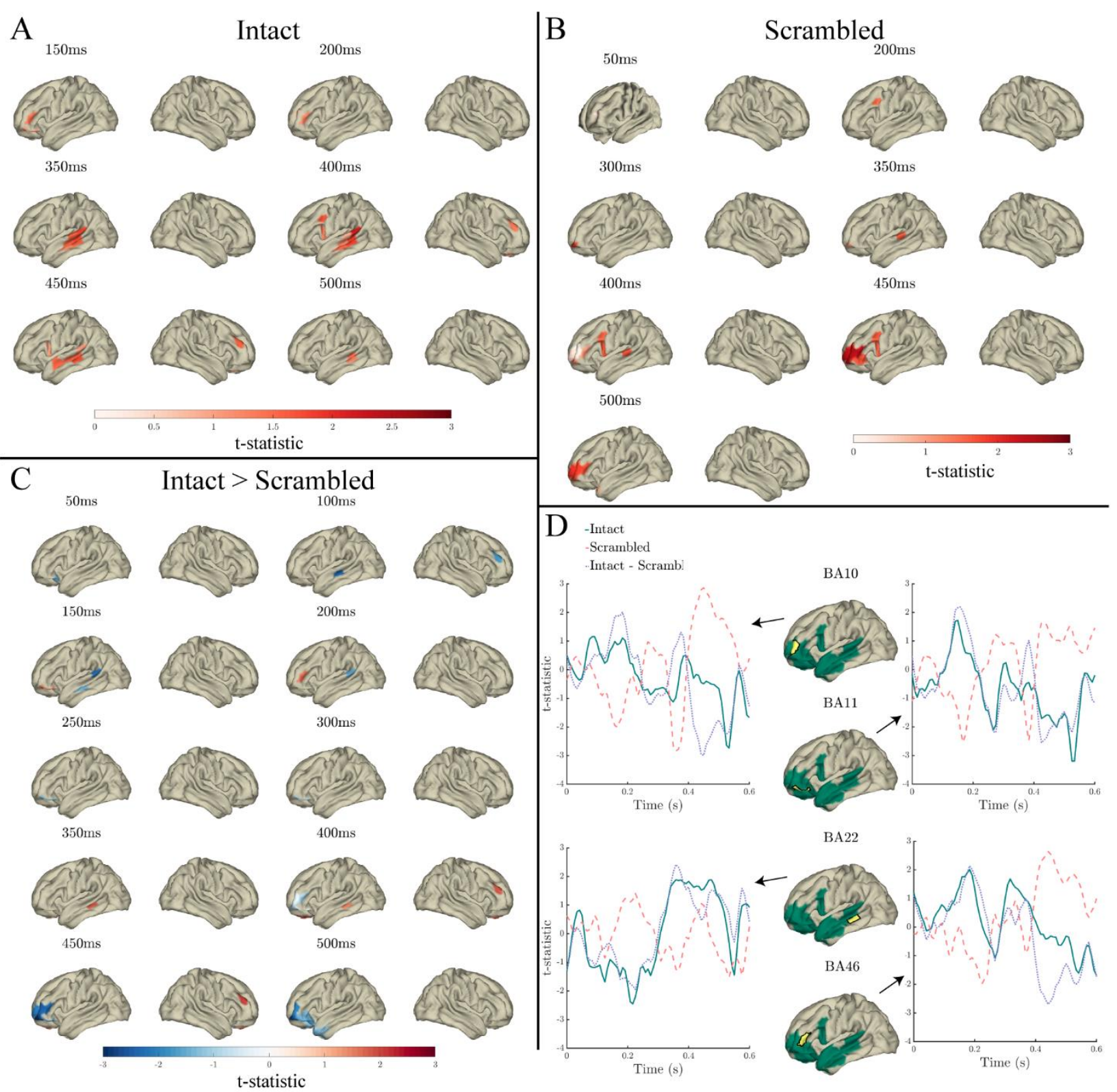

t-statistic

Figure 7. Effects of the lexical frequency $\times$ index interaction in the response to content words:

631 Surface plots of T-statistics (averaged over 50ms time windows centred at the indicated latencies,

632 for visualisation) quantifying the difference in variance explained by lexical frequency $\times$ index 633 interaction, beyond that explained by lexical frequency ( $\log 10$ transformed), index, and length in intact sentence compared to random permutation models (panel $\mathrm{A} ; p<.05$ one-sided, uncorrected), scrambled sentence compared to random permutation models (panel $\mathrm{B} ; p<.05$ one-sided, uncorrected), and intact compared to scrambled sentence models (panel C; $p<.05$ two-sided, uncorrected). Parcels for which no time point was significant during the $50 \mathrm{~ms}$ time bin are masked. Panel D: Time courses of T-statistics for intact (solid green line) and scrambled (dashed red line) sentence models compared to random permutation models, and intact compared to scrambled sentence models (dotted purple line) for subparcels of BA10, BA11, BA22 and BA46 (highlighted in yellow on adjacent surface plots). ROIs entered into statistical analyses are illustrated as green 642 shaded areas on surface plots. 


\section{Lexical frequency $\times$ Surprisal}

644 The interaction between lexical frequency and surprisal significantly predicted MEG signal

645 variance, beyond the main effects of lexical frequency, surprisal and word length, from 275-

$646392 \mathrm{~ms}$, starting in a frontal parcel (BA11), and spreading to anterior temporal parcels from $283 \mathrm{~ms}$

647 (BA38), and further throughout temporal (BA21/BA22) and frontal (BA44/BA46) parcels, from

$648292 \mathrm{~ms}$ and 308ms respectively. Effects peaked at around 350ms (see Fig 8 panel A-B; corrected $649 \quad p^{<.05)}$.

650 
A
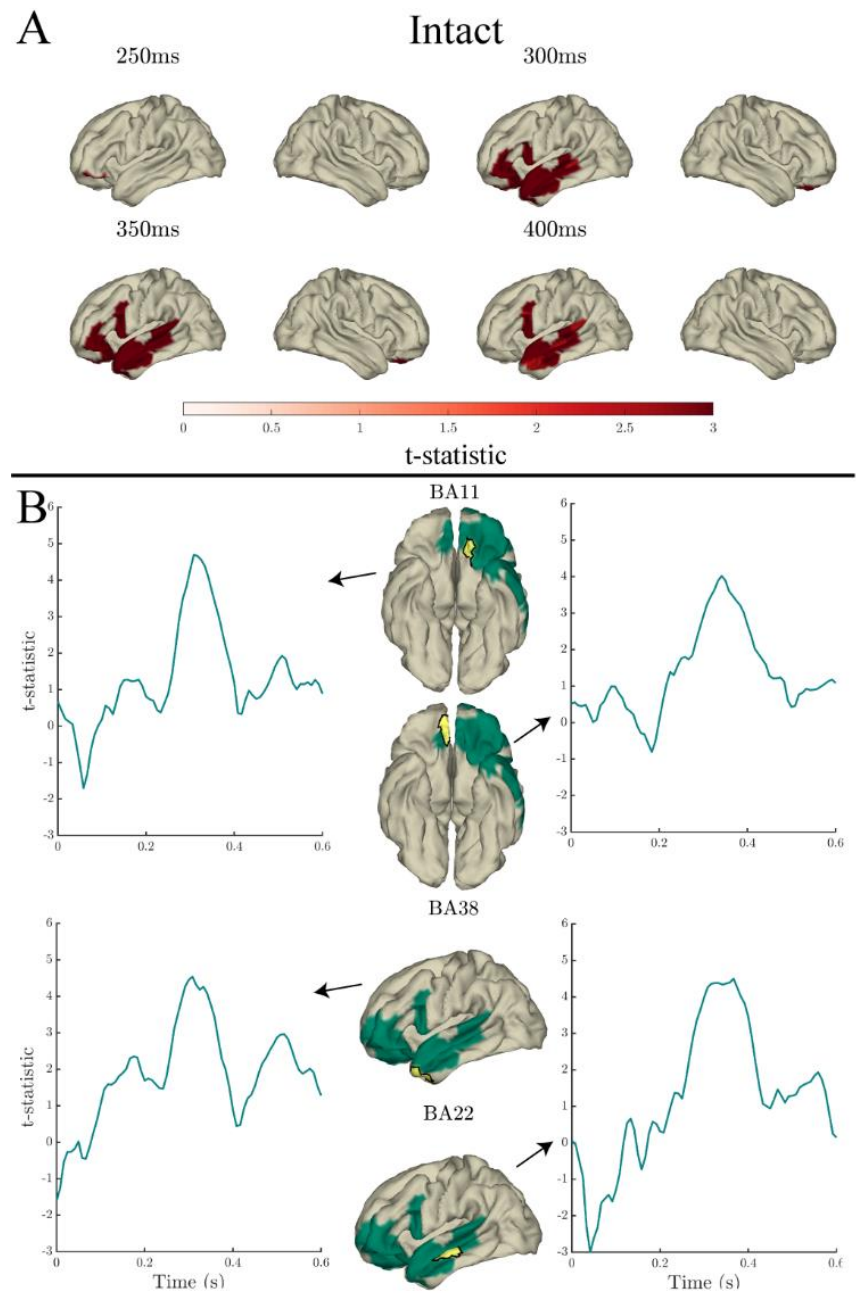

653 Figure 8 . Effects of the lexical frequency $\times$ surprisal interaction in the response to content words: 654 Surface plots of T-statistics (averaged over 50ms time windows centred at the indicated latencies, 655 for visualisation) quantifying the difference in variance explained by lexical frequency $\times$ surprisal 656 interaction, beyond that explained by lexical frequency (log10 transformed), surprisal $(\log 10$ 657 transformed), and word length, in intact sentence compared to random permutation models (panel $658 \mathrm{~A} ; p<.05$ one-sided, corrected). Parcels for which no time point was significant during the 50ms 659 time bin are masked. Panel B: Time courses of T-statistics for intact sentence models compared to 660 random permutation models, for subparcels of BA11 (left hemisphere), BA11 (right hemisphere), 661 BA38 and BA22 (highlighted in yellow on adjacent surface plots). ROIs entered into statistical 662 analyses are illustrated as green shaded areas on surface plots. 
665 Beyond the variance explained by the main effects of entropy, lexical frequency, and length, the

666 lexical frequency $\times$ entropy interaction explained additional variance from 200-600ms, starting in

667 anterior temporal (BA38/BA21/BA22) and frontal (BA11) parcels, spreading further throughout

668 frontal parcels (BA10/BA44) from 250ms/330ms (respectively) and posteriorly through middle

669 and superior temporal cortex (see Fig 9; uncorrected $p<.05$ ). The effect of the interaction peaked

670 at around $350 \mathrm{~ms}$, and again at approximately $500 \mathrm{~ms}$.

$\mathrm{A}_{200 \mathrm{~ms}}$

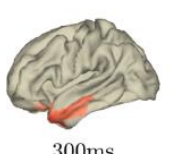

$300 \mathrm{~ms}$

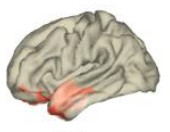

$400 \mathrm{~ms}$

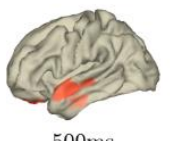

$500 \mathrm{~ms}$
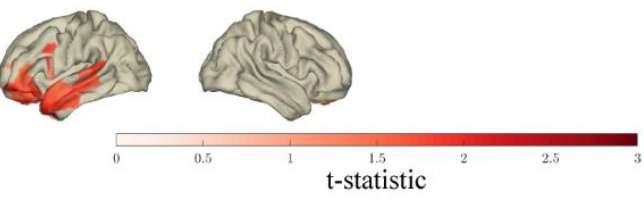

Intact
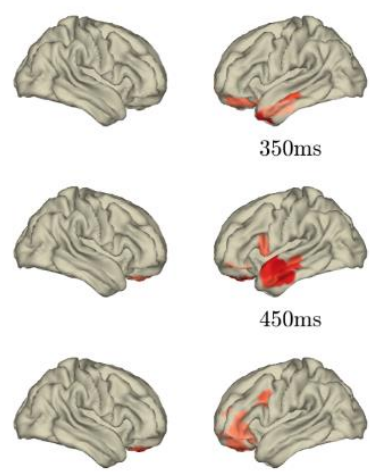

$450 \mathrm{~ms}$

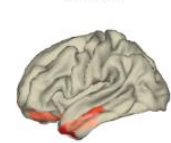

$0 \mathrm{~ms}$

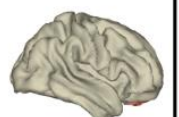

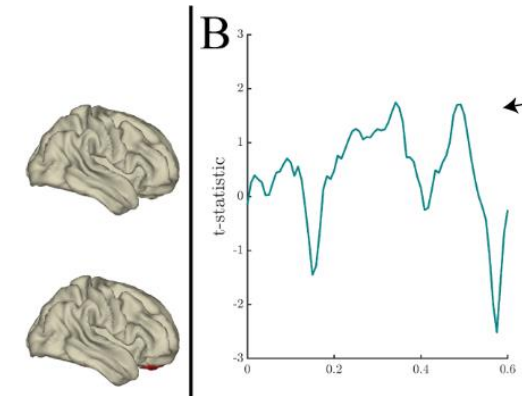

BA44
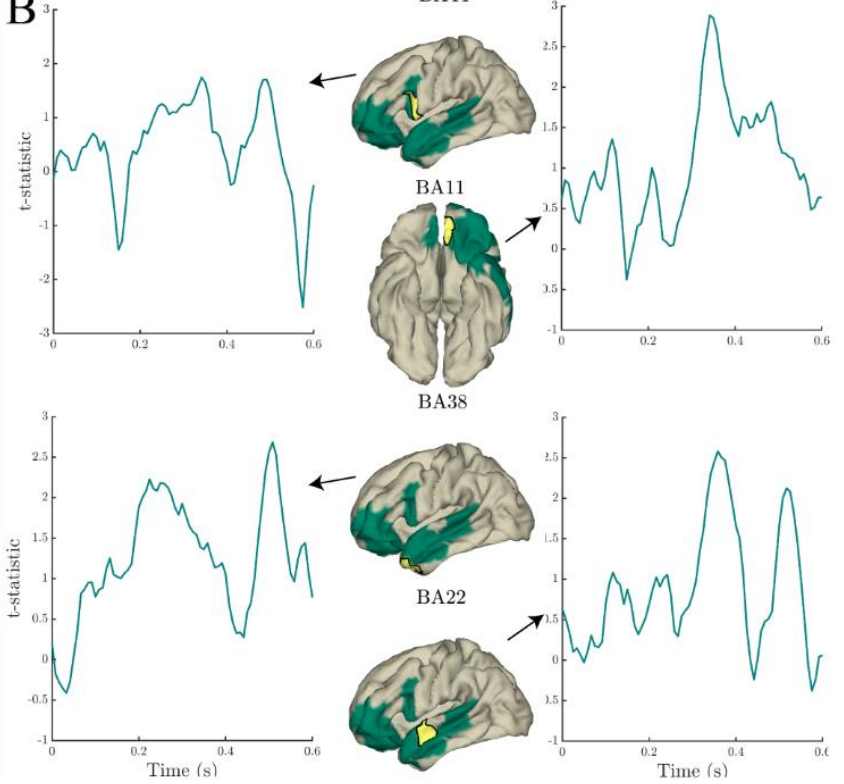

672

673

674

675

676

677

678

679

680

681

Figure 9. Effects of the lexical frequency $\times$ entropy interaction in the response to content words: Surface plots of T-statistics (averaged over 50ms time windows centred at the indicated latencies, for visualisation) quantifying the difference in variance explained by lexical frequency $\times$ entropy interaction, beyond that explained by lexical frequency (log10 transformed), entropy, and word length, in intact sentence compared to random permutation models (panel A; $p<.05$ one-sided, uncorrected). Parcels for which no time point was significant during the $50 \mathrm{~ms}$ time bin are masked. Panel B: Time courses of T-statistics for intact sentences models compared to random permutation models, for subparcels of BA44, BA11, BA38 and BA22 (highlighted in yellow on adjacent surface plots). ROIs entered into statistical analyses are illustrated as green shaded areas on surface plots. 


\section{Discussion}

684 During sentence reading, the brain processes individual words at a remarkable speed. Such fast 685 processing is not only facilitated and affected by the word's frequency of occurrence within a given 686 language (Calvo \& Meseguer, 2002; Inhoff \& Rayner, 1986; Rayner \& Duffy, 1986; Rubenstein 687 et al., 1970), but also by the word's context, brought about by semantic and syntactic constraints 688 imposed by preceding words (Calvo \& Meseguer, 2002; Staub et al., 2015; Van Petten \& Kutas, 689 1990). There is a well-documented discrepancy between the electrophysiological and eye-tracking 690 literature as to whether frequency and context have additive or interactive effects on processing 691 (Kretzschmar et al., 2015). It is unclear whether word frequency influences processing when the

692 input is predictable. The current work aimed to better define the spatiotemporal dynamics of the 693 effects of lexical frequency and predictability on word processing, establish to what extent lexical 694 frequency and predictability independently influence word processing, and to what extent they 695 interact. To this end, we performed state-of-the-art analysis of a large and well-balanced MEG 696 dataset, combining spatiotemporal hyperalignment with cross-validated encoding model 697 comparisons. This allowed us to go beyond the more traditional approaches that use event-related 698 averaging or generalized linear models, thus being able to infer effects based on the brain's 699 response to individual words.

700 We found that the MEG signal reflects the lexical frequency of individual words (here content 701 words) throughout the analysis time window beyond effects of predictability, in a network 702 expanding from occipital cortex throughout the left temporal and inferior frontal regions of the 703 language network. Index, surprisal, and entropy additionally each significantly predicted the MEG 704 signal. All comparisons were made while controlling for each alternative predictor, and word 705 length. There were significant but focal differences between intact and scrambled sentences in the 
706 effects of lexical frequency, surprisal and entropy. In contrast to these focal differences, the effect

707 of index differed extensively in intact compared to scrambled sentences. Thus, out of the analysed

708 predictors, only the effect of index was greatly influenced by the sentential context in which words

709 were presented (i.e. intact/scrambled sentences). These findings highlight that the word processing

710 mechanisms reflected by index are dependent on the preceding context, whereas the processing

711 mechanisms underlying lexical frequency and surprisal remain largely the same regardless of the

712 degree of sentential context. Finally, only the interaction between lexical frequency and surprisal

713 survived multiple comparisons correction (in ventromedial PFC and anterior temporal lobe), and

714 not the interaction between lexical frequency and entropy, nor between lexical frequency and

715 index. Although the index $\times$ lexical frequency interaction effect was not significant under a

716 conservative multiple comparisons correction scheme, an inspection of the uncorrected results

717 uncovered an interesting pattern. Namely, both left temporal and frontal cortical activity seemed

718 to be influenced by the interaction, yet the latency at which this occurred was flipped across

719 conditions. While, in intact sentences, the interaction was expressed more strongly at early time

720 points in frontal areas and only later in temporal areas, this pattern was reversed for scrambled

721 sentences. Importantly, on inspection of both the corrected and uncorrected results, the interactions

722 between lexical frequency and our metrics quantifying predictability show an initial peak between

$723 \quad 150-250 \mathrm{~ms}$. Given that the average fixation duration lasts $\sim 200 \mathrm{~ms}$, any processing related to eye

724 movement decisions must occur prior to this time window (Sereno \& Rayner, 2003). Our findings

725 tentatively support that lexical frequency and predictability do not interact robustly until around

$726150 \mathrm{~ms}$ or later, which could explain why eye movement studies display a purely additive effect of

727 these variables, in contrast to the robust interaction observed across electrophysiological studies.

728 In the following paragraphs we discuss the results in more detail. 


\subsection{Lexical frequency}

730 Overall, lexical frequency was encoded in the MEG signal to a similar extent in intact and

731 scrambled sentences. This effect was widespread, both in space and time, and thus suggests that

732 lexical frequency generically affects the brain response, likely reflecting less effortful processing

733 of high compared to low frequency words. These findings help to close the gap between the

734 electrophysiological and eye tracking literature, by providing evidence that frequency indeed

735 influences word processing independently of prediction. In contrast to the eye tracking literature,

736 electrophysiological studies have previously found that, during word processing, effects of lexical

737 frequency disappear with increased context (Dambacher et al., 2006; Payne et al., 2015; Sereno et

738 al., 2019; Van Petten \& Kutas, 1990).

739 Although our findings differ from Fruchter et al. (2015), who found that word frequency explained

740 no additional variance in the MEG signal after word onset beyond that explained by predictability,

741 our results are consistent with the overall findings from the paper. Specifically, the authors

742 presented evidence that, rather than reflecting a baseline level of predictability, lexical frequency

743 influenced lexical access itself, as the frequency of the predicted word affected the

744 electrophysiological response in the MTG prior to seeing the word (i.e. in response to the highly

745 constraining word).

746 In the current data, effects of lexical frequency were observed after controlling for predictability

747 prior to $100 \mathrm{~ms}$ in occipital cortex. Such an early response in visual processing regions likely

748 reflects an influence of word frequency on identification of the word form. To measure the extent

749 that these early effects could be explained by the frequency of lower level sublexical properties of

750 the word form, rather than the frequency of the lexeme, we conducted an additional analysis of

751 lexical frequency while controlling for bigram and trigram letter frequency, as well as all other 
752 predictors (see Methods section 2.7). The results of this analysis are presented in the

753 supplementary material (Fig SM1). Here it can be seen that the overall effect of word frequency

754 remained the same as compared to when these variables were not controlled for (see Fig 3).

755 Although lexical frequency explained variance in a reduced number of occipital and occipito-

756 temporal parcels while controlling for the words' lower level visual characteristics, compared to

757 the results presented in Fig 3, an effect of lexical frequency was still observed in visual cortex at

758 around 100ms. Lexical frequency therefore seems to influence early visual processing, beyond

759 effects of the frequency of lower level properties of the word form.

760 The effect of lexical frequency progressively moved anteriorly through temporal and frontal cortex

761 throughout word processing, supporting that lexical frequency influences multiple stages of word

762 processing, such as lexical access and integration with the sentential context. These findings are

763 in line with the EZ model of word reading (Reichle, Pollatsek, \& Rayner, 2012), which proposes

764 that word frequency and predictability independently affect both early (word form recognition)

765 and late (lexical access/integration/compositional) stages of processing. Comparing intact and

766 scrambled sentences, frequency was encoded in the MEG signal earlier in intact than scrambled

767 sentences in the STG and MTG. Given the association of the MTG with lexical-semantic

768 processing (Friederici, 2012; Hagoort, 2017) and the location of the primary auditory cortex and

769 auditory association areas on the STG, the current results suggest that lexical frequency facilitates

770 aspects of semantic and phonological processing earlier when the word is presented in a coherent

771 sentence than when presented in a scrambled sentence. Moreover, significantly more variance was

772 explained in intact compared to scrambled sentences at $267 \mathrm{~ms}$ in a single dorsolateral PFC parcel

773 (BA46), an area thought to be involved in executive control during language processing (Hagoort,

$7742003,2013,2017)$. 


\subsection{Sentential context and predictability}

776 In line with previous literature (Armeni, Willems, van den Bosch, \& Schoffelen, 2019; Hultén,

777 Schoffelen, Uddén, Lam, \& Hagoort, 2019; Schuster et al., 2020), the word-by-word association

778 between the MEG signals and the increasingly constrained context (i.e. index), and metrics 779 quantifying (the results of) prediction, presented itself with different spatiotemporal dynamics.

780 These will be discussed in the following paragraphs.

$781 \quad$ Index

782 Index explained a significant portion of variance in the MEG signal during the entire critical

783 window in both intact and scrambled sentences. Moreover, index predicted the MEG signal

784 significantly more in intact than scrambled sentences, predominantly in anterior temporal and

785 frontal cortex. This latter finding illustrates that it is the progressing sentential context that affects

786 word processing in these regions, rather than more domain-general properties that correlate with

787 index, such as working memory demands. The anterior temporal lobe has been associated with

788 conceptual representations (Peelen \& Caramazza, 2012; Pylkkänen, 2019; Ralph, Jefferies,

789 Patterson, \& Rogers, 2017; Rice, Lambon Ralph, \& Hoffman, 2015) and syntactic structure

790 building (Brennan et al., 2012; Brennan \& Pylkkänen, 2017), the latter of which is engaged more

791 when words are presented in intact compared to scrambled sentences. The greater influence of

792 index in intact compared to scrambled sentences in the inferior frontal gyrus is consistent with the

793 notion of unification, the integration of lexical items within the wider semantic and syntactic

794 context as the sentence unfolds (Hagoort, 2005, 2013).

795 In line with earlier work (Schuster et al., 2020), index was encoded in the MTG and angular gyrus

796 in intact sentences. No such effect was observed in these regions for scrambled sentences, although

797 the latter qualitative difference was not significant when directly contrasting conditions. Given the 
association between MTG activity and lexical-semantic processing (Friederici, 2012; Hagoort,

799 2017), the effect in MTG could reflect the build-up of richer semantic representations as coherent

800 sentences progress, more so than during the progression of scrambled sentences. The absence of

801 an effect of index in scrambled sentences in the angular gyrus may be consistent with the view that

802 this region is a hub to integrate different types of information extracted by various parts of the

803 language network (Binder \& Desai, 2011; Hagoort, 2003, 2019). Although, the precise roles of the

804 angular gyrus and the anterior temporal lobe in integrating conceptual information are still

805 currently debated (Binder \& Desai, 2011; Hagoort, 2019; Matchin, Liao, Gaston, \& Lau, 2019;

806 Pylkkänen, 2019; Ralph et al., 2017). In contrast to unfolding well-formed sentences, scrambled

807 sentences lack syntactic structure, and therefore do not permit for a meaningful integration of

808 structural cues with, for instance, lexico-semantic information.

\section{Surprisal}

810 We estimated surprisal and entropy using corpus-based statistics, using a tri-gram model on the

811 individual intact and scrambled sentences. Consistent with our expectations, surprisal was overall

812 larger in scrambled sentence words (see Fig 1). Yet, aside from subtle differences between intact

813 and scrambled sentences, as discussed below, the overall spatiotemporal characteristics of MEG

814 signal variance explained by surprisal, on top of the other predictors, was similar between

815 conditions. One tentative explanation for this could be that the inclusion of the index predictor in

816 the 'baseline model' already accounted for a large part of signal variance (albeit to different

817 degrees across conditions), causing the additional information provided by surprisal values to be

818 less distinctive across conditions. The word-by-word fluctuations in surprisal explained

819 widespread, predominantly left-lateralized, brain signals, irrespective of condition. This suggests

820 a relation between our operationalisation of surprisal on the one hand, and more automatic ease- 
821 of-integration related processes on the other hand. Although care was taken to scramble sentences

822 in a way so as no more than three consecutive words could be syntactically combined, there is

823 evidence that combinatorial processes are robust to local word swaps (Mollica et al., 2020). In the

824 current data, surprisal seems to reflect the same underlying combinatorial processes in scrambled

825 and intact sentences, reflecting the ease-of-integration.

826 A direct statistical comparison across conditions showed some very focal and short-lived

827 differences. Apart from a very early time window, at around 50ms in the MTG, there was a

828 difference around 400-450ms in orbitofrontal and MTG parcels. It is often difficult to determine

829 whether observed effects of surprisal result from participants predicting the upcoming linguistic

830 input, or from more probable words being easier to integrate (Pickering \& Gambi, 2018; Willems,

831 Frank, Nijhof, Hagoort, \& van den Bosch, 2016). While the early effect of surprisal that we observe

832 here is likely related to predictive mechanisms, the later MEG signatures might equally be caused

833 by hindered integration. Surprisal was encoded in the MEG signal in temporal cortex prior to

$834100 \mathrm{~ms}$ (in the sentence condition only), which has previously been argued to imply that some

835 linguistic information about a word has been pre-activated - here constrained by the previous two

836 words - given that bottom-up lexical retrieval could not yet have taken place (Pickering \& Gambi,

837 2018). Although the precise timing of lexical access of written words is currently debated, it is

838 thought that sub-lexical characteristics and the word form have been processed by $\sim 100 \mathrm{~ms}$ and

839 morphemic processing and lexical access of the lemma occurs between 150-170ms (Grainger \&

840 Holcomb, 2009; Hauk et al., 2006; Lewis, Solomyak, \& Marantz, 2011; Pulvermüller, Shtyrov, \&

841 Hauk, 2009; Sereno \& Rayner, 2003; Woollams, 2015). Such timings speak to a pre-activation

842 account of the early effects of surprisal in the temporal cortex here. Sentence context may influence

843 the timing of lexical retrieval through prediction mechanisms (Fruchter et al., 2015). In contrast, 
844 the later effects of surprisal at 400-450ms in orbitofrontal and MTG parcels could result from

845 either integrative or predictive processes. Although the orbitofrontal cortex (situated in the

846 ventromedial PFC) has previously been sensitive to predictability of both linguistic information

847 (Hofmann et al., 2014) and more generally (Nobre, Coull, Frith, \& Mesulam, 1999), the

848 ventromedial PFC has also been associated with higher level combinatorial processes (Brennan \&

849 Pylkkänen, 2008, 2010; Pylkkänen, 2008, 2019, 2020; Pylkkänen, Martin, McElree, \& Smart,

850 2009; Pylkkänen \& McElree, 2007), in line with an integrative account of the later effect of

851 surprisal here.

852 Entropy

853 Entropy quantifies the uncertainty of the upcoming linguistic content (Pickering \& Gambi, 2018;

854 Willems et al., 2016). Entropy significantly predicted the MEG signal in both intact and scrambled

855 sentences. Notably, the spatial and temporal extent of significant effects were much smaller than

856 those of the other predictors. Here, entropy was encoded in early occipital cortical activity, both

857 in intact and scrambled sentences. Additionally, in sentences, entropy effects were observed in left

858 frontal cortex around 300ms, and in inferior temporal cortex around 450ms. Effects of prediction

859 in occipital parcels during early time points have previously been used as evidence to support the

860 notion that an active prediction of word form is employed by the brain (Dikker, Rabagliati, Farmer,

861 \& Pylkkänen, 2010; Pickering \& Gambi, 2018). Rather than directly reflecting prediction, entropy

862 quantifies the uncertainty about upcoming words (here based on the prior two words). Prediction

863 of upcoming words was not possible in the scrambled sentence condition. Participants have been

864 shown to quickly adapt their predictive behaviour to the predictability of the linguistic content of

865 the current context (Bosker, van Os, Does, \& van Bergen, 2019; Heyselaar, Peeters, \& Hagoort,

866 2020; Thacker, Chambers, \& Graham, 2018). It therefore seems unlikely that, when reading 
867 scrambled sentences, participants still pre-activated word forms that would usually be likely

868 candidates to follow in a sentence. An alternative explanation for the early occipital cortical

869 activity here is that, under uncertainty of upcoming linguistic input, more weight is placed on

870 bottom-up (as opposed to top-down) signal, and more resources are allocated to visual processing.

871 In contrast to the more generic interpretation of early entropy effects in visual cortical areas, the

872 later sentence-specific effect in inferior temporal cortex could indeed reflect predictive processing

873 of the word form. This region, often referred to as the visual word form area, is likely to receive

874 top-down signals containing linguistic information about a word (Price \& Devlin, 2011; Sharoh et

875 al., 2019).

876 Entropy presented with a markedly different pattern of results compared to the other prediction

877 metrics, in that only several focal groups of parcels during narrow time points survived multiple

878 comparisons correction. It is evident from the time courses in Fig 6 that the encoding of the MEG

879 signal was temporally less consistent for the entropy models compared to the models presented in

880 Figs 3-5. Similarly, Schuster et al. (2020) found no effect of predictability (entropy) in the

881 haemodynamic response when conducting a whole-brain analysis, and effects were found only in 882 an ROI analysis.

\section{4.3. Interactions between lexical frequency and predictability}

884 In line with previous work (Alday et al., 2017; Dambacher et al., 2006; Fruchter et al., 2015; Payne

885 et al., 2015; Sereno et al., 2019; Van Petten \& Kutas, 1990), we investigated the interaction

886 between lexical frequency and metrics quantifying prediction, including index (both within and

887 across individual conditions), surprisal and entropy (in sentences only). Here we add to the

888 previous literature by investigating the spatiotemporal dynamics of the interaction in more detail

889 in comparison to previous reports (Fruchter et al., 2015). Using a strict multiple comparisons 
890 correction scheme, we found evidence of an interaction only between lexical frequency and 891 surprisal, and not between lexical frequency and index, nor lexical frequency and entropy. The 892 latter two findings seem to concur with the eye-tracking literature, which has found an additive 893 effect of lexical frequency and predictability on fixation durations (Kennedy et al., 2013; 894 Kretzschmar et al., 2015; Staub, 2015; Staub \& Benatar, 2013). Yet, the lexical frequency $\times$ 895 surprisal interaction results are in line with the electrophysiological literature, in which effects of 896 lexical frequency on word processing are reduced with increased predictability (Dambacher et al., 897 2012; Dambacher et al., 2006; Kretzschmar et al., 2015; Sereno et al., 2003; Sereno et al., 2019). 898 Furthermore, partially supporting the aforementioned electrophysiological literature, an analysis 899 of the nominally thresholded data revealed a spatially similar pattern of results of the entropy 900 interaction as compared to the significant interaction with surprisal (corrected $p<.05$ ), in addition 901 to some interesting condition-specific dynamics of the lexical frequency $\times$ index interaction. 902 Finally, all three interactions first peaked between 150-250ms, suggesting that these variables 903 could additively influence early stages of word processing prior to $150 \mathrm{~ms}$, but interact during later, 904 post-lexical stages of word processing. Such findings help to explain why, in contrast to the 905 electrophysiological literature, only an additive effect of these variables has been observed in the 906 eye tracking literature. Given that an average fixation duration lasts 200ms (Rayner, 1986), eye 907 movement decisions should only be influenced by information obtained in early stages of word 908 processing (Sereno \& Rayner, 2003).

909 Firstly, lexical frequency interacted with surprisal and entropy in frontal (predominantly in BA11) 910 and anterior temporal parcels, the interaction being strongest at around 350ms. Both the anterior 911 temporal lobe and BA11 have been proposed to be involved in combinatorial processes during 912 sentence comprehension, the former in semantic (Binder \& Desai, 2011; Brennan \& Pylkkänen, 
913 2017; Hagoort, 2019; Matchin, Liao, et al., 2019; Pylkkänen, 2019; Ralph et al., 2017) or syntactic

914 (Brennan et al., 2012; Brennan \& Pylkkänen, 2017) integration, and the latter in higher level

915 compositional processing and inferring implicit meanings (Brennan \& Pylkkänen, 2008, 2010;

916 Pylkkänen, 2008, 2019, 2020; Pylkkänen et al., 2009; Pylkkänen \& McElree, 2007). The frequency

917 of a word may therefore become less relevant to its integration within the higher level sentential

918 meaning when the same word is highly predictable. Although we do not report the direction of the

919 interaction here (see Section 4.4. Limitations and future work), previous reports have consistently

920 shown that the effect of frequency on word processing diminishes with increased predictability,

921 and the benefits of predictability on word processing are enhanced for low compared to high

922 frequency words (Dambacher et al., 2012; Dambacher et al., 2006; Fruchter et al., 2015; Hofmann

923 et al., 2014; Kretzschmar et al., 2015; Sereno et al., 2003; Sereno et al., 2019). For example, similar

924 to the current results, an interaction between lexical frequency and predictability was found in

925 orbitofrontal cortex (encompassed in BA11) by Hofmann et al. (2014), who found stronger brain

926 responses to disconfirmed predictions for only low and not high frequency words.

927 The interaction between lexical frequency and index displayed some intriguing dynamics in time

928 and space across conditions (despite not surviving multiple comparisons corrections). In left

929 temporal parcels (BA21/BA22/BA38), including the MTG, the interaction explained more

930 variance in scrambled than intact sentences at early time points, and in intact compared to

931 scrambled sentences in a later time window. The later $(350-500 \mathrm{~ms})$ temporal cortex effect is

932 consistent with previous electrophysiological literature that has averaged over central-parietal

933 sensors in an N400 time window, as the interaction explained more variance in coherent sentences

934 than in scrambled sentences. Specifically, earlier work has shown that the effect of frequency on

935 the N400 diminishes with increased word position, in intact sentences but not scrambled sentences 
936 (Payne et al., 2015), eliciting the conclusion that lexical frequency no longer influences word 937 processing when there is increased context. An interaction between word frequency and 938 predictability in the left MTG is also consistent with the findings of Fruchter et al. (2015), who 939 found an effect of frequency here only for words of low and not high predictability. One 940 mechanism through which this could occur is through the pre-activation of semantic features 941 associated with the lexical item, or pre-activation of the lexical item itself, so that processing low 942 frequency words is no longer as difficult compared to high frequency words.

943 In frontal parcels (BA10/BA11), more variance was explained by the interaction in intact 944 compared to scrambled sentences in an early time window, and in scrambled compared to intact 945 sentences in a later time window. Greater ventromedial PFC (BA11) recruitment has previously 946 been observed in sentences compared to word-lists more generally (Brennan \& Pylkkänen, 2012) 947 and, as discussed above, is thought to be involved in interpreting higher level sentence meanings 948 (Brennan \& Pylkkänen, 2008, 2010; Pylkkänen, 2008, 2019, 2020; Pylkkänen et al., 2009; 949 Pylkkänen \& McElree, 2007). BA10, on the other hand, has been associated with encoding 950 semantic relationships (Bunge et al., 2009; Frankland \& Greene, 2020; Knowlton et al., 2012; 951 Ramnani \& Owen, 2004). Both higher level compositional processing and forming semantic 952 relationships could be expected to occur earlier in intact compared to scrambled sentences. Overall, 953 the difference between intact and scrambled sentences in the interaction between lexical frequency 954 and index seems to occur in the time that these factors interact, rather than in the presence of an 955 interaction.

956 Current models of word reading do not yet account for the effects observed in the current data, 957 together with the aforementioned eye tracking and electrophysiological literature. The EZ-Reader 958 model (Reichle et al., 2012), and more recent Uber-Reader model (Veldre, Yu, Andrews, \& 
959 Reichle, 2020) of word reading, propose that lexical frequency and predictability independently

960 influence both early (L1/identification of the word form) and late (L2/lexical access/semantic

961 processing/integration) stages of word processing. While we provide confirmatory evidence that

962 lexical frequency and predictability indeed influence both early and late stages of word processing

963 independently, we also show that they interact during later stages of word processing. Models of

964 word reading could therefore benefit from incorporating these additional findings. Although we

965 do not quantify the direction of the interaction here, previous reports have robustly demonstrated

966 that the effect of lexical frequency is reduced for highly predictable words (compared to

967 unpredictable words), and the effect of predictability is greater for low than high frequency words

968 (Dambacher et al., 2012; Dambacher et al., 2006; Fruchter et al., 2015; Hofmann et al., 2014;

969 Kretzschmar et al., 2015; Sereno et al., 2003; Sereno et al., 2019).

\section{4.4. Limitations and future work}

971 A limitation of the current work is that words were presented word-by-word, causing the

972 stimulation to be externally paced. Yet, it is well known that in more naturalistic settings the

973 reading pace is determined by the reader, where eye movement and fixation behaviour is in part

974 the result of prediction related processes (Rayner \& Well, 1996). Indeed, there is evidence to

975 suggest that predictability facilitates processing before a word is fixated, while the word is within

976 parafoveal view (Balota, Pollatsek, \& Rayner, 1985; Staub, 2015; Staub \& Goddard, 2019).

977 Furthermore, self-paced reading paradigms have demonstrated that fixation durations of the

978 current word are influenced by the properties of the preceding word (Dambacher \& Kliegl, 2007;

979 Kliegl, Nuthmann, \& Engbert, 2006). Predictive processes may be engaged at different latencies

980 or to a different extent in natural reading compared to the current paradigm, due to their interaction

981 with the executive control of eye movements. Future work should aim to investigate whether the 
982 observed spatiotemporal dynamics of the effects of lexical frequency and predictability on the

983 MEG signal hold during naturalistic reading (see Brennan \& Pylkkänen, 2017 for an investigation

984 into naturalistic word reading with MEG).

985 A further limitation of the current investigation is that we did not report the direction of the 986 interaction between lexical frequency and our metrics quantifying predictability. As the variables

987 in the models were highly correlated (see Fig 1), it is not possible to meaningfully interpret the 988 beta weights from the models. Instead, we used a model comparison scheme to quantify the 989 additional variance explained by each regressor, beyond that already explained by all other 990 regressors (see Methods section 2.7 for details). Given that the direction of the interaction is robust 991 across numerous previous reports, a lack of directionality in the current results does not greatly 992 hinder the interpretation of our results. Moreover, by comparing intact and scrambled sentences, 993 we were able to report the degree to which the strength of the interaction changed with and without 994 sentential context. In doing so, we replicated previous findings of a stronger interaction in intact 995 compared to scrambled sentences during a typical N400 time window in the temporal cortex. 996 However, we additionally showed that the direction of this higher order interaction reversed in the 997 frontal cortex during the same time window, and in the temporal cortex in an earlier time window. 998 Future work with more carefully controlled stimuli could aim to replicate these results in a ROI 999 analysis.

\section{4.5. Conclusions}

1002 We provide evidence to support that frequency and contextual constraints have identifiable effects 1003 on multiple stages of word-by-word processing, from early visual and lexical retrieval to later 1004 integration and unification processes. Largely similar spatiotemporal effects across both intact and 
1005 scrambled sentences suggest that lexical frequency generally affects how fast and effortful 1006 processing is, independently from ongoing predictive processes.

1007 Only the interaction between lexical frequency and surprisal survived our conservative multiple 1008 comparisons corrections (in anterior temporal and frontal cortex), and not the interaction between 1009 lexical frequency and entropy, nor between lexical frequency and index. Although we found no 1010 significant effect of a lexical frequency $\times$ index interaction - consistent with the additive effects of 1011 these variables typically observed in the eye-tracking literature - an uncorrected analysis revealed 1012 some interesting spatiotemporal dynamics. Namely, the effect of the interaction was reversed in 1013 time and space in intact sentences compared to scrambled sentences. In the MTG, which is 1014 associated with lexical-semantic processing, the interaction explained more variance in scrambled 1015 sentences than intact sentences in an early time window, and in intact sentences than scrambled 1016 sentences in a later time window. The latter is consistent with the frequency $\times$ index interaction 1017 that is typically observed in the N400 time window in intact sentences but not scrambled sentences 1018 (Payne et al., 2015). In orbitofrontal and ventromedial PFC cortex, which have previously been 1019 associated with forming higher level semantic relationships and inferring implicit meanings, the 1020 interaction explained more variance in intact sentences than scrambled sentences at early time 1021 points, but in scrambled sentences than intact sentences in a later time window. Finally, we provide 1022 evidence to suggest that lexical frequency and predictability may independently influence early 1023 and late stages of word processing, but also interact during later stages of word processing. Our 1024 findings may contribute to improved models of word reading, which do not yet fully account for 1025 effects of predictability in the current results, nor in previous work (Staub \& Goddard, 2019). 


\section{Acknowledgements}

1027 We would like to thank Alessandro Lopopolo for computing the corpus-derived lexical 1028 characteristics of lexical frequency, surprisal and entropy for the current stimulus set. This work 1029 was supported by The Netherlands Organisation for Scientific Research (NWO Vidi: 864.14.011).

\section{References}

1032 Alday, P. M., Schlesewsky, M., \& Bornkessel-Schlesewsky, I. (2017). Electrophysiology Reveals 1033

Arana, S., Marquand, A., Hultén, A., Hagoort, P., \& Schoffelen, J.-M. (2020). Sensory ModalityIndependent Activation of the Brain Network for Language. The Journal of Neuroscience,

Armeni, K., Willems, R. M., van den Bosch, A., \& Schoffelen, J.-M. (2019). Frequency-specific brain dynamics related to prediction during language comprehension. Neuroimage, 198, 283-295. doi:https://doi.org/10.1016/j.neuroimage.2019.04.083

1042 Balota, D. A., Pollatsek, A., \& Rayner, K. (1985). The interaction of contextual constraints and parafoveal visual information in reading. Cognitive Psychology, 17(3), 364-390.

1045 Binder, J. R., \& Desai, R. H. (2011). The neurobiology of semantic memory. Trends in Cognitive Sciences, 15(11), 527-536. doi:https://doi.org/10.1016/j.tics.2011.10.001

1047 Bosker, H. R., van Os, M., Does, R., \& van Bergen, G. (2019). Counting 'uhm's: How tracking 1048 the distribution of native and non-native disfluencies influences online language 
comprehension. Journal of Memory and Language, 106, 189-202. doi:https://doi.org/10.1016/j.jml.2019.02.006

Brennan, J., Nir, Y., Hasson, U., Malach, R., Heeger, D. J., \& Pylkkänen, L. (2012). Syntactic structure building in the anterior temporal lobe during natural story listening. Brain and Language, 120(2), 163-173. doi:https://doi.org/10.1016/j.bandl.2010.04.002

Brennan, J., \& Pylkkänen, L. (2008). Processing events: Behavioral and neuromagnetic correlates of Aspectual Coercion. Brain and Language, 106(2), 132-143. doi:https://doi.org/10.1016/j.band1.2008.04.003

Brennan, J., \& Pylkkänen, L. (2010). Processing psych verbs: Behavioural and MEG measures of two different types of semantic complexity. Language and Cognitive Processes, 25(6), 777-807. doi:10.1080/01690961003616840

Brennan, J., \& Pylkkänen, L. (2012). The time-course and spatial distribution of brain activity associated with sentence processing. Neuroimage, 60(2), 1139-1148. doi:https://doi.org/10.1016/j.neuroimage.2012.01.030

Brennan, J., \& Pylkkänen, L. (2017). MEG Evidence for Incremental Sentence Composition in the Anterior Temporal Lobe. Cognitive Science, 41(S6), 1515-1531. doi:https://doi.org/10.1111/cogs.12445

Brysbaert, M. (2019). How many words do we read per minute? A review and meta-analysis of reading rate. Journal of Memory and Language, 109. doi:https://doi.org/10.1016h/j.jml.2019.104047

Bunge, S. A., Helskog, E. H., \& Wendelken, C. (2009). Left, but not right, rostrolateral prefrontal cortex meets a stringent test of the relational integration hypothesis. Neuroimage, 46(1), 338-342. doi:https://doi.org/10.1016/j.neuroimage.2009.01.064 
1072 Calvo, M. G., \& Meseguer, E. (2002). Eye Movements and Processing Stages in Reading: Relative 1073 Contribution of Visual, Lexical, and Contextual Factors. The Spanish Journal of 1074 Psychology, 5(1), 66-77. doi:10.1017/S1138741600005849

1075 Catani, M., Allin, M. P. G., Husain, M., Pugliese, L., Mesulam, M. M., Murray, R. M., \& Jones, 1076

D. K. (2007). Symmetries in human brain language pathways correlate with verbal recall. Proceedings of the National Academy of Sciences, 104(43), 17163-17168. doi:10.1073/pnas.0702116104

Chee, M. W. L., Hon, N. H. H., Caplan, D., Lee, H. L., \& Goh, J. (2002). Frequency of Concrete Words Modulates Prefrontal Activation during Semantic Judgments. Neuroimage, 16(1), 259-268. doi:https://doi.org/10.1006/nimg.2002.1061

Chen, Y., Davis, M. H., Pulvermüller, F., \& Hauk, O. (2015). Early Visual Word Processing Is Flexible: Evidence from Spatiotemporal Brain Dynamics. J Cogn Neurosci, 27(9), 17381751. doi:10.1162/jocn_a_00815

Crepaldi, D., Rastle, K., Coltheart, M., \& Nickels, L. (2010). 'Fell'primes 'fall', but does 'bell'prime 'ball'? Masked priming with irregularly-inflected primes. Journal of Memory and Language, 63(1), 83-99.

Dambacher, M., Dimigen, O., Braun, M., Wille, K., Jacobs, A. M., \& Kliegl, R. (2012). Stimulus onset asynchrony and the timeline of word recognition: Event-related potentials during sentence reading. $\quad$ Neuropsychologia, $50(8)$, $1852-1870$. doi:https://doi.org/10.1016/j.neuropsychologia.2012.04.011

Dambacher, M., \& Kliegl, R. (2007). Synchronizing timelines: Relations between fixation durations and N400 amplitudes during sentence reading. Brain Research, 1155, 147-162. doi:https://doi.org/10.1016/j.brainres.2007.04.027 
1095 Dambacher, M., Kliegl, R., Hofmann, M., \& Jacobs, A. M. (2006). Frequency and predictability

1096 effects on event-related potentials during reading. Brain Research, 1084(1), 89-103. 1097 doi:https://doi.org/10.1016/j.brainres.2006.02.010

1098 de Cheveigné, A., Di Liberto, G. M., Arzounian, D., Wong, D. D. E., Hjortkjær, J., Fuglsang, S., 1099 \& Parra, L. C. (2019). Multiway canonical correlation analysis of brain data. Neuroimage, 1100 186, 728-740. doi:https://doi.org/10.1016/j.neuroimage.2018.11.026

1101 Dikker, S., Rabagliati, H., Farmer, T. A., \& Pylkkänen, L. (2010). Early Occipital Sensitivity to 1102 Syntactic Category Is Based on Form Typicality. Psychological Science, 21(5), 629-634. $1103 \quad$ doi:10.1177/0956797610367751

1104 Engbert, R., Nuthmann, A., Richter, E. M., \& Kliegl, R. (2005). SWIFT: A Dynamical Model of 1105 Saccade Generation During Reading. Psychological Review, 112(4), 777-813. 1106 doi:10.1037/0033-295X.112.4.777

1107 Frankland, S. M., \& Greene, J. D. (2020). Two Ways to Build a Thought: Distinct Forms of 1108 Compositional Semantic Representation across Brain Regions. Cerebral Cortex, 30(6), 1109

1110 Friederici, A. D. (2009). Pathways to language: fiber tracts in the human brain. Trends in Cognitive 1111 Sciences, 13(4), 175-181. doi:https://doi.org/10.1016/j.tics.2009.01.001

1112 Friederici, A. D. (2012). The cortical language circuit: from auditory perception to sentence 1113 comprehension. Trends in Cognitive Sciences, 16(5), 262-268. 1114 doi:https://doi.org/10.1016/j.tics.2012.04.001

1115 Fruchter, J., Linzen, T., Westerlund, M., \& Marantz, A. (2015). Lexical Preactivation in Basic 1116 Linguistic Phrases. Journal of Cognitive Neuroscience, 27(10), 1912-1935. 1117 doi:10.1162/jocn_a_00822 
1118 Glasser, M. F., \& Rilling, J. K. (2008). DTI Tractography of the Human Brain's Language 1119 Pathways. Cerebral Cortex, 18(11), 2471-2482. doi:10.1093/cercor/bhn011

1120 Grainger, J., \& Holcomb, P. J. (2009). Watching the Word Go by: On the Time-course of 1121 Component Processes in Visual Word Recognition. Language and Linguistics Compass, 3(1), 128-156. doi:10.1111/j.1749-818X.2008.00121.x

1123 Hagoort, P. (2003). How the brain solves the binding problem for language: a neurocomputational model of syntactic processing. Neuroimage, 20, S18-S29. doi:https://doi.org/10.1016/j.neuroimage.2003.09.013

1126 Hagoort, P. (2005). On Broca, brain, and binding: a new framework. Trends in Cognitive Sciences, 9(9), 416-423. doi:https://doi.org/10.1016/j.tics.2005.07.004

1128 Hagoort, P. (2013). MUC (Memory, Unification, Control) and beyond. Frontiers in Psychology, 4(416). doi:10.3389/fpsyg.2013.00416

1130 Hagoort, P. (2017). The core and beyond in the language-ready brain. Neuroscience \& Biobehavioral Reviews.

1132 Hagoort, P. (2019). The meaning-making mechanism(s) behind the eyes and between the ears. Philosophical Transactions of the Royal Society B: Biological Sciences, 375(1791).

1135 Hagoort, P., Baggio, G., \& Willems, R. M. (2009). Semantic unification. In The cognitive neurosciences, 4th ed. (pp. 819-836): MIT press.

1137 Hauk, O., Patterson, K., Woollams, A., Watling, L., Pulvermüller, F., \& Rogers, T. T. (2006). [Q:] 1138 When Would You Prefer a SOSSAGE to a SAUSAGE? [A:] At about 100 msec. ERP 1139 Correlates of Orthographic Typicality and Lexicality in Written Word Recognition. $1140 \quad$ Journal of Cognitive Neuroscience, 18(5), 818-832. doi:10.1162/jocn.2006.18.5.818 
1141 Heyselaar, E., Peeters, D., \& Hagoort, P. (2020). Do we predict upcoming speech content in 1142 naturalistic environments? Language, Cognition and Neuroscience, 1-22. 1143 doi: $10.1080 / 23273798.2020 .1859568$

1144 Hofmann, M. J., Dambacher, M., Jacobs, A. M., Kliegl, R., Radach, R., Kuchinke, L., . . . 1145 Herrmann, M. J. (2014). Occipital and orbitofrontal hemodynamics during naturally paced reading: An fNIRS study. Neuroimage, 94, 193-202.

Huettig, F. (2015). Four central questions about prediction in language processing. Brain Research, 1626, 118-135. doi:https://doi.org/10.1016/j.brainres.2015.02.014

1150 Hultén, A., Schoffelen, J.-M., Uddén, J., Lam, N. H. L., \& Hagoort, P. (2019). How the brain makes sense beyond the processing of single words - An MEG study. Neuroimage, 186,

1153 Inhoff, A. W., \& Rayner, K. (1986). Parafoveal word processing during eye fixations in reading: Effects of word frequency. Perception \& Psychophysics, 40(6), 431-439.

1155 Kennedy, A., Pynte, J., Murray, W. S., \& Paul, S.-A. (2013). Frequency and predictability effects in the Dundee Corpus: An eye movement analysis. The Quarterly Journal of Experimental

Kliegl, R., Nuthmann, A., \& Engbert, R. (2006). Tracking the mind during reading: The influence of past, present, and future words on fixation durations. Journal of experimental psychology: general, 135(1), 12-35. doi:10.1037/0096-3445.135.1.12

1161 Knowlton, B. J., Morrison, R. G., Hummel, J. E., \& Holyoak, K. J. (2012). A neurocomputational system for relational reasoning. Trends in Cognitive Sciences, 16(7), 373-381. doi:https://doi.org/10.1016/j.tics.2012.06.002 
1164 Kresse, L., Kirschner, S., Dipper, S., \& Belke, E. (2012). Towards exploring the specific influences 1165 of wordform frequency, lemma frequency and OLD20 on visual word recognition and reading aloud. Lexical Resources in Psycholinguistic Research, 3, 9.

1167 Kretzschmar, F., Schlesewsky, M., \& Staub, A. (2015). Dissociating Word Frequency and

1171 Kutas, M., \& Federmeier, K. D. (2011). Thirty Years and Counting: Finding Meaning in the N400 1172 Component of the Event-Related Brain Potential (ERP). Annual Review of Psychology, 62(1), 621-647. doi:10.1146/annurev.psych.093008.131123

1174 Lam, N. H. L., Schoffelen, J. M., Udden, J., Hulten, A., \& Hagoort, P. (2016). Neural activity during sentence processing as reflected in theta, alpha, beta, and gamma oscillations. Neuroimage, 142, 43-54. doi:10.1016/j.neuroimage.2016.03.007

Lau, E. F., \& Namyst, A. (2019). fMRI evidence that left posterior temporal cortex contributes to N400 effects of predictability independent of congruity. Brain and Language, 199, 104697. doi:https://doi.org/10.1016/j.bandl.2019.104697

Lau, E. F., Phillips, C., \& Poeppel, D. (2008). A cortical network for semantics: (de)constructing the N400. Nature Reviews Neuroscience, 9(12), 920-933. doi:10.1038/nrn2532

Levy, R. (2008). Expectation-based syntactic comprehension. Cognition, 106(3), 1126-1177. doi:https://doi.org/10.1016/j.cognition.2007.05.006

Lewis, G., Solomyak, O., \& Marantz, A. (2011). The neural basis of obligatory decomposition of suffixed words. Brain and Language, 118(3), 118-127. doi:https://doi.org/10.1016/j.bandl.2011.04.004 
1187 Matchin, W., Brodbeck, C., Hammerly, C., \& Lau, E. (2019). The temporal dynamics of structure 1188 and content in sentence comprehension: Evidence from fMRI-constrained MEG. Human Brain Mapping, 40(2), 663-678. doi:10.1002/hbm.24403

1190 Matchin, W., Liao, C.-H., Gaston, P., \& Lau, E. (2019). Same words, different structures: An fMRI investigation of argument relations and the angular gyrus. Neuropsychologia, 125, 116128. doi:https://doi.org/10.1016/j.neuropsychologia.2019.01.019

1193 Mollica, F., Siegelman, M., Diachek, E., Piantadosi, S. T., Mineroff, Z., Futrell, R., . . Fedorenko, 1194 E. (2020). Composition is the Core Driver of the Language-selective Network. Neurobiology of Language, 1(1), 104-134.doi:10.1162/nol_a_00005

1196 Nobre, A. C., Coull, J. T., Frith, C. D., \& Mesulam, M. M. (1999). Orbitofrontal cortex is activated during breaches of expectation in tasks of visual attention. Nature Neuroscience, 2(1), 11-

Nolte, G. (2003). The magnetic lead field theorem in the quasi-static approximation and its use for 1200 magnetoencephalography forward calculation in realistic volume conductors. Physics in Medicine \& Biology, 48(22), 3637.

1204 12. doi: $10.1038 / 4513$

Peelen, M. V., \& Caramazza, A. (2012). Conceptual Object Representations in Human Anterior 1206 Temporal Cortex. The Journal of Neuroscience, 32(45), 15728-15736. doi:10.1523/jneurosci.1953-12.2012 
1208 Penolazzi, B., Hauk, O., \& Pulvermuller, F. (2007). Early semantic context integration and lexical 1209 access as revealed by event-related brain potentials. Biological Psychology, 74(3), 3741210 388. doi:10.1016/j.biopsycho.2006.09.008

1211 Pickering, M. J., \& Gambi, C. (2018). Predicting While Comprehending Language: A Theory and 1212 Review. Psychological Bulletin, 144(10), 1002-1044. doi:10.1037/bul0000158

1213 Price, C. J., \& Devlin, J. T. (2011). The Interactive Account of ventral occipitotemporal 1214 contributions to reading. Trends in Cognitive Sciences, 15(6), 246-253.

1216 Pulvermüller, F., Shtyrov, Y., \& Hauk, O. (2009). Understanding in an instant: Neurophysiological doi:https://doi.org/10.1016/j.tics.2011.04.001

1219 Pylkkänen, L. (2008). Mismatching Meanings in Brain and Behavior. Language and Linguistics 1220

1221 Pylkkänen, L. (2019). The neural basis of combinatory syntax and semantics. Science, 366(6461), 1222

1223 Pylkkänen, L. (2020). Neural basis of basic composition: what we have learned from the 1224 1225 1226 1227 1228

1229 Pylkkänen, L., \& McElree, B. (2007). An MEG Study of Silent Meaning. Journal of Cognitive 1230 Neuroscience, 19(11), 1905-1921. doi:10.1162/jocn.2007.19.11.1905 
1231 Ralph, M. A. L., Jefferies, E., Patterson, K., \& Rogers, T. T. (2017). The neural and computational 1232 bases of semantic cognition. Nature Reviews Neuroscience, $18,42$. 1233 doi:10.1038/nrn.2016.150

1234 https://www.nature.com/articles/nrn.2016.150\#supplementary-information

1235 Ramnani, N., \& Owen, A. M. (2004). Anterior prefrontal cortex: insights into function from 1236 anatomy and neuroimaging. Nature Reviews Neuroscience, 5(3), 184-194. 1237 doi: $10.1038 /$ nrn1343

1238 Rayner, K. (1986). Eye movements and the perceptual span in beginning and skilled readers. 1239 Journal of Experimental Child Psychology, 41(2), 211-236. doi:10.1016/00220965(86)90037-8

1241 Rayner, K., \& Duffy, S. A. (1986). Lexical complexity and fixation times in reading: Effects of word frequency, verb complexity, and lexical ambiguity. Memory \& Cognition, 14(3), 191-

1244 Rayner, K., \& Well, A. D. (1996). Effects of contextual constraint on eye movements in reading: 1245 A further examination. Psychonomic Bulletin \& Review, 3(4), 504-509.

1246 Reichle, E. D., Pollatsek, A., \& Rayner, K. (2012). Using E-Z Reader to simulate eye movements 1247 in nonreading tasks: A unified framework for understanding the eye-mind link. $1248 \quad$ Psychological Review, 119(1), 155-185. doi:10.1037/a0026473

1249 Reichle, E. D., Rayner, K., \& Pollatsek, A. (2003). The E-Z Reader model of eye-movement 1250 control in reading: Comparisons to other models. Behavioral and Brain Sciences, 26(4), 1251 445-476. doi:10.1017/S0140525X03000104 
1252 Rice, G. E., Lambon Ralph, M. A., \& Hoffman, P. (2015). The Roles of Left Versus Right Anterior 1253 Temporal Lobes in Conceptual Knowledge: An ALE Meta-analysis of 97 Functional 1254 Neuroimaging Studies. Cerebral Cortex, 25(11), 4374-4391. doi:10.1093/cercor/bhv024

1255 Rubenstein, H., Garfield, L., \& Millikan, J. A. (1970). Homographic entries in the internal lexicon. 1256

1258 Schäfer, R., \& Bildhauer, F. (2012). Building large corpora from the web using a new efficient 1259 Journal of Verbal Learning and Verbal Behavior, 9(5), 487-494. doi:https://doi.org/10.1016/S0022-5371(70)80091-3 tool chain. Paper presented at the LREC.

Schoffelen, J.-M., Hultén, A., Lam, N., Marquand, A. F., Uddén, J., \& Hagoort, P. (2017). Frequency-specific directed interactions in the human brain network for language. Proceedings of the National Academy of Sciences, 114(30), 8083-8088. doi:10.1073/pnas.1703155114

1264 Schoffelen, J.-M., Oostenveld, R., Lam, N. H. L., Uddén, J., Hultén, A., \& Hagoort, P. (2019). A 1265 204-subject multimodal neuroimaging dataset to study language processing. Scientific Data, 6(1), 17. doi:10.1038/s41597-019-0020-y

Schuster, S., Hawelka, S., Himmelstoss, N. A., Richlan, F., \& Hutzler, F. (2020). The neural correlates of word position and lexical predictability during sentence reading: evidence from fixation-related fMRI. Language, Cognition and Neuroscience, 35(5), 613-624. doi:10.1080/23273798.2019.1575970

1273

Sereno, S. C., Brewer, C. C., \& O'Donnell, P. J. (2003). Context Effects in Word Recognition:Evidence for Early Interactive Processing. Psychological Science, 14(4), 328333. doi:10.1111/1467-9280.14471 
1274 Sereno, S. C., Hand, C. J., Shahid, A., Mackenzie, I. G., \& Leuthold, H. (2019). Early EEG 1275 correlates of word frequency and contextual predictability in reading. Language Cognition and Neuroscience. doi:10.1080/23273798.2019.1580753

1277 Sereno, S. C., \& Rayner, K. (2003). Measuring word recognition in reading: eye movements and 1278 event-related potentials. Trends in Cognitive Sciences, 7(11), 489-493. doi:https://doi.org/10.1016/j.tics.2003.09.010

Sharoh, D., van Mourik, T., Bains, L. J., Segaert, K., Weber, K., Hagoort, P., \& Norris, D. G. (2019). Laminar specific fMRI reveals directed interactions in distributed networks during language processing. Proceedings of the National Academy of Sciences, 116(42), 2118521190. doi:10.1073/pnas.1907858116

Smith, M. E., \& Halgren, E. (1987). Event-related potentials during lexical decision: effects of repetition, word frequency, pronounceability, and concreteness. Electroencephalogr Clin Neurophysiol Suppl, 40, 417-421.

Staub, A. (2015). The Effect of Lexical Predictability on Eye Movements in Reading: Critical Review and Theoretical Interpretation. Language and Linguistics Compass, 9(8), 311-327. doi:10.1111/lnc3.12151

Staub, A., \& Benatar, A. (2013). Individual differences in fixation duration distributions in reading. Psychonomic Bulletin \& Review, 20(6), 1304-1311. doi:10.3758/s13423-0130444-x

Staub, A., \& Goddard, K. (2019). The Role of Preview Validity in Predictability and Frequency Effects on Eye Movements in Reading. Journal of Experimental Psychology-Learning Memory and Cognition, 45(1), 110-127. doi:10.1037/xlm0000561 
1296 Staub, A., Grant, M., Astheimer, L., \& Cohen, A. (2015). The influence of cloze probability and 1297 item constraint on cloze task response time. Journal of Memory and Language, 82, 1-17. 1298 doi:10.1016/j.jml.2015.02.004

1299 Strijkers, K., Bertrand, D., \& Grainger, J. (2015). Seeing the same words differently: the time 1300 course of automaticity and top-down intention in reading. J Cogn Neurosci, 27(8), 15421301 1551.doi:10.1162/jocn_a_00797

1302 Thacker, J. M., Chambers, C. G., \& Graham, S. A. (2018). When it is apt to adapt: Flexible 1303 1304 reasoning guides children's use of talker identity and disfluency cues. Journal of Experimental Child Psychology, 167, 314-327.

1306 Van Den Bosch, A., \& Berck, P. (2009). Memory-Based Machine Translation and Language 1307 Modeling. Prague Bull. Math. Linguistics, 91, 17-26.

1308 Van Petten, C., \& Kutas, M. (1990). Interactions between sentence context and word 1309 frequencyinevent-related brainpotentials. Memory \& Cognition, 18(4), 380-393. doi:10.3758/bf03197127

1311 Van Veen, B. D., van Drongelen, W., Yuchtman, M., \& Suzuki, A. (1997). Localization of brain 1312 electrical activity via linearly constrained minimum variance spatial filtering. IEEE Transactions on Biomedical Engineering, 44(9), 867-880. doi:10.1109/10.623056

1314 Veldre, A., Yu, L., Andrews, S., \& Reichle, E. D. (2020). Towards a complete model of reading: 1315 Simulating lexical decision, word naming, and sentence reading with Über-Reader. 1316 Proceedings of the 42nd Annual Conference of the Cognitive Science Society, 151-157. 1317 Retrieved from https://hdl.handle.net/2123/22990 
1318 Willems, R. M., Frank, S. L., Nijhof, A. D., Hagoort, P., \& van den Bosch, A. (2016). Prediction

1319 During Natural Language Comprehension. Cerebral Cortex, 26(6), 2506-2516. 1320 doi:10.1093/cercor/bhv075

1321 Woollams, A. M. (2015). Lexical is as lexical does: computational approaches to lexical 1322 representation. Language, Cognition and Neuroscience, 30(4), 395-408. doi:10.1080/23273798.2015.1005637

1324 


\section{Lexical frequency and sentence context influence the brain's response to single words Supplementary material \\ Eleanor Huizeling, Sophie Arana, Peter Hagoort, Jan Mathijs Schoffelen}

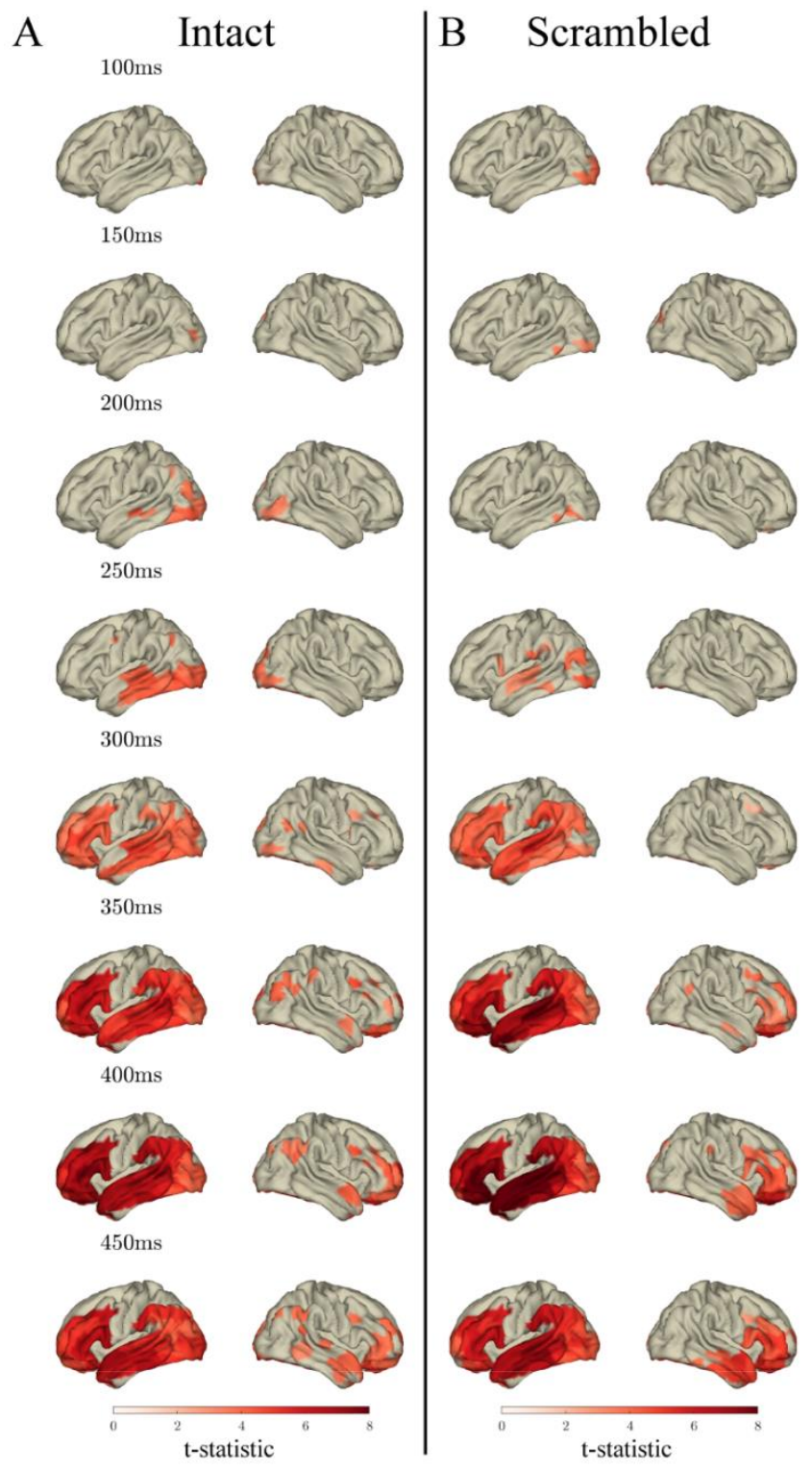

Figure SM1. Effects of lexical frequency in the response to content words: Surface plots of Tstatistics (averaged over 50ms time windows centred at the indicated latencies, for visualisation) quantifying the difference in variance explained by lexical frequency (log 10 transformed), beyond that explained by index, surprisal (log10 transformed), entropy, length, bigram letter frequency ( $\log 10$ transformed) and trigram letter frequency (log10 transformed) in intact sentence compared to random permutation models (panel A; $p<.05$ one-sided, corrected) and scrambled sentence compared to random permutation models (panel B; $p<.05$ one-sided, corrected). Parcels for which no time point was significant during the $50 \mathrm{~ms}$ time bin are masked. 


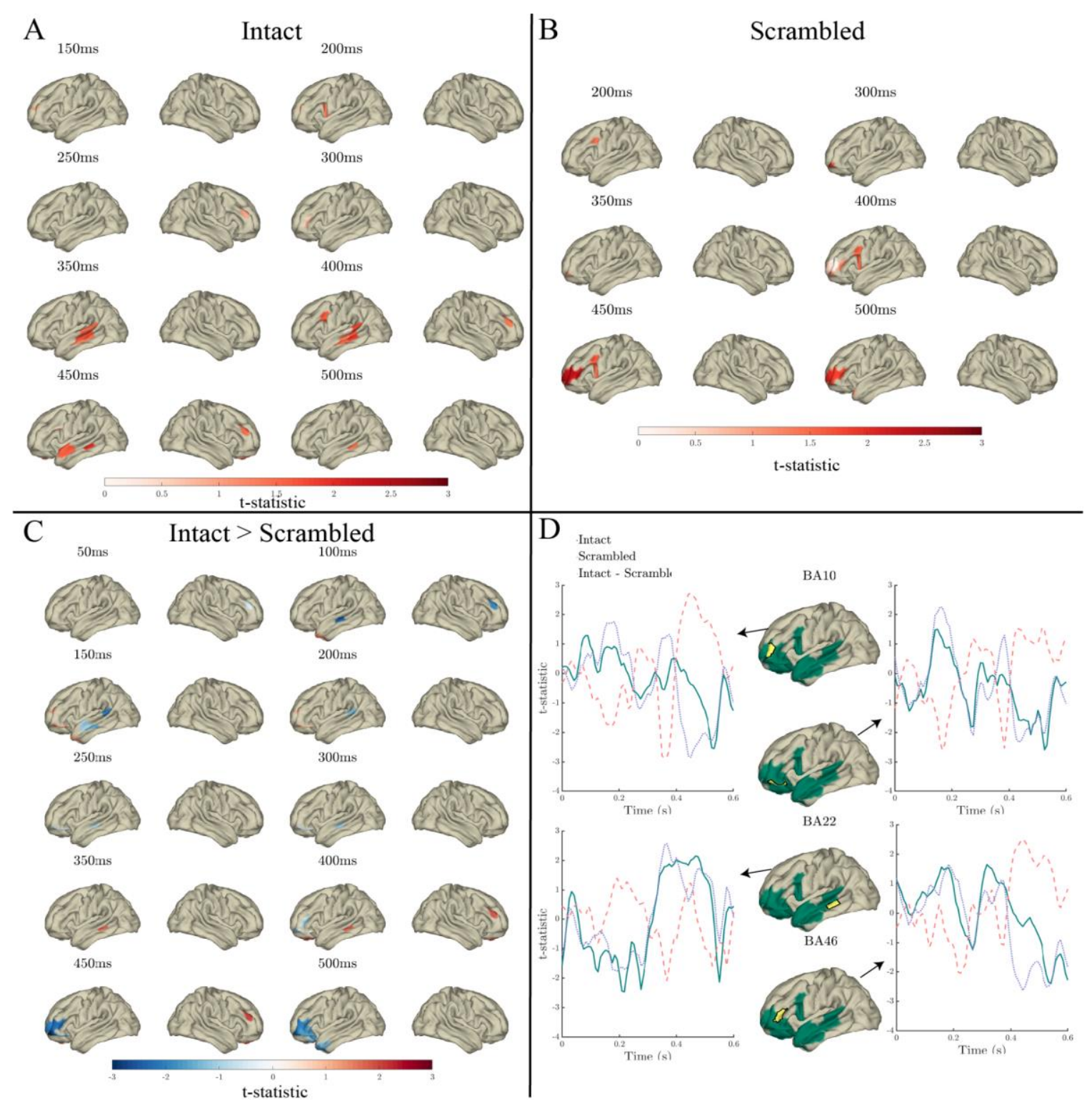

Figure SM2. Effects of the lexical frequency $\times$ index interaction in the response to content words: Surface plots of T-statistics (averaged over $50 \mathrm{~ms}$ time windows centred at the indicated latencies, for visualisation) quantifying the difference in variance explained by lexical frequency $\times$ index interaction, beyond that explained by lexical frequency ( $\log 10$ transformed), index, length, bigram letter frequency (log10 transformed) and trigram letter frequency (log10 transformed) in intact sentence compared to random permutation models (panel $\mathrm{A} ; p<.05$ one-sided, uncorrected), scrambled sentence compared to random permutation models (panel $\mathrm{B} ; p<.05$ one-sided, uncorrected), and intact compared to scrambled sentence models (panel $\mathrm{C} ; p<.05$ two-sided, corrected). Parcels for which no time point was significant during the $50 \mathrm{~ms}$ time bin are masked. Panel D: Time courses of T-statistics for intact (solid green line) and scrambled (dashed red line) sentence models compared to random permutation models, and intact compared to scrambled sentence models (dotted purple line) for subparcels of BA10, BA11 BA22 and BA46 (highlighted in yellow on adjacent surface plots). ROIs entered into statistical analyses are illustrated as green shaded areas on surface plots. 


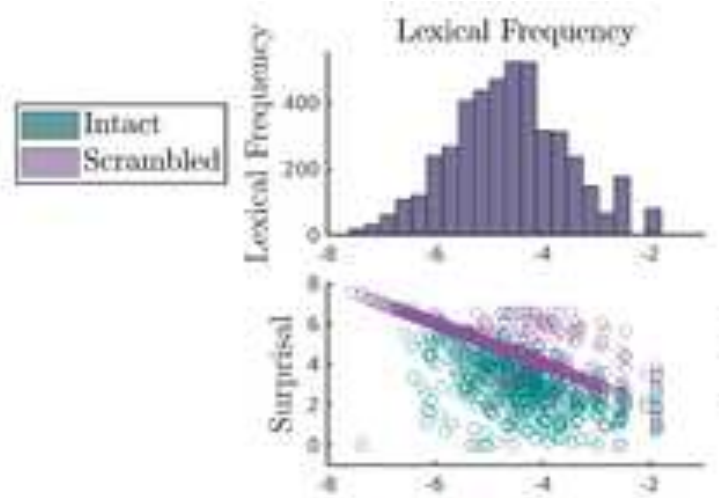

Surprisal
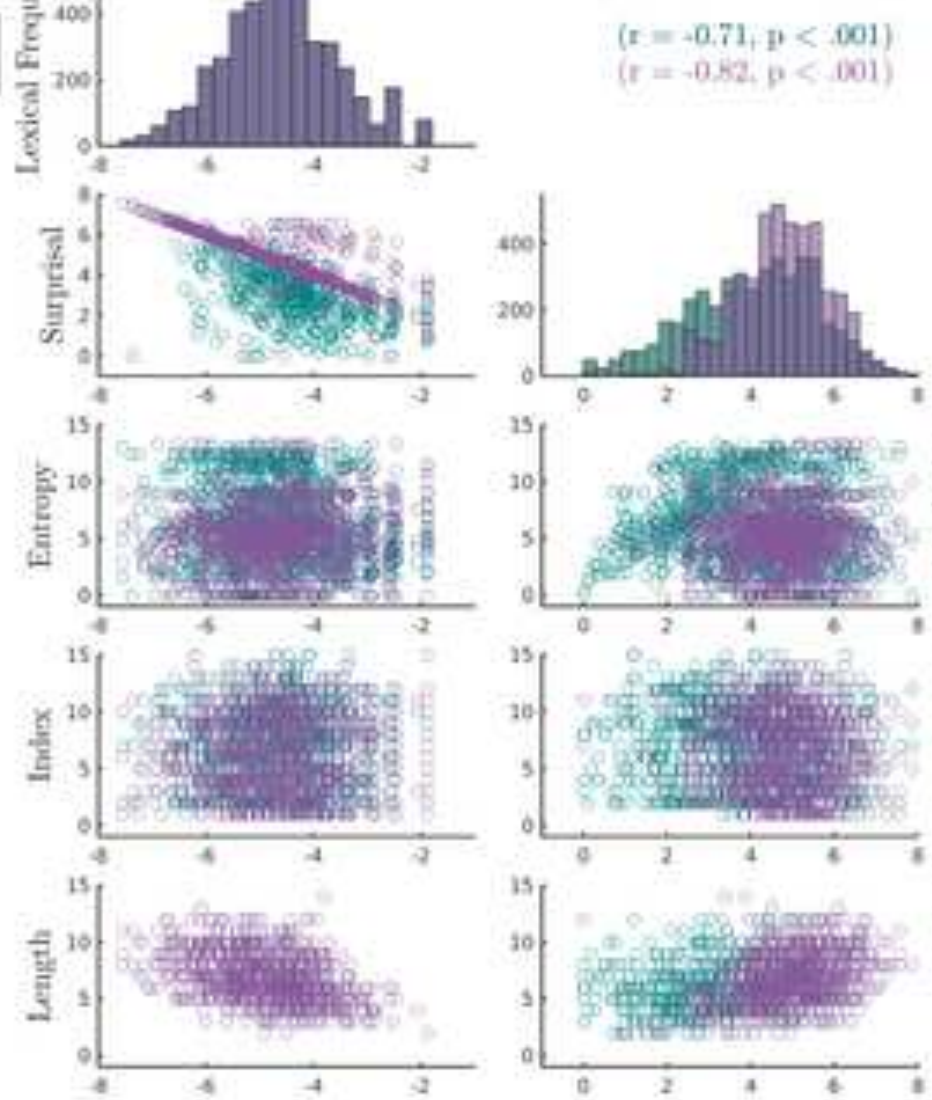
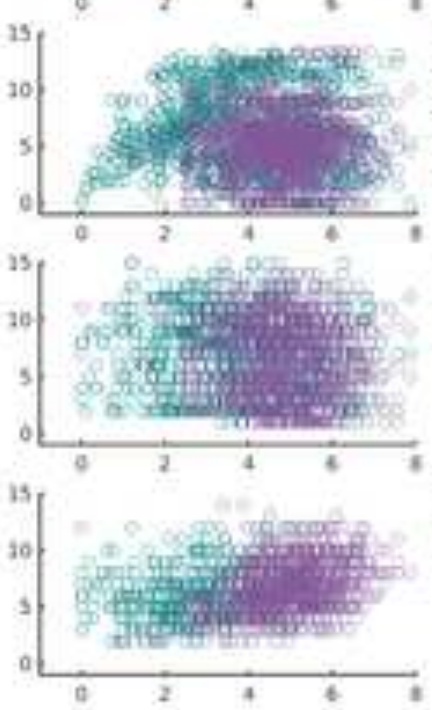

Entropy

$$
\begin{aligned}
& (r=-0.15, \mathrm{p}<.001) \\
& (r=0.10, \mathrm{p}<.001)
\end{aligned}
$$

$(x=0.02, p=0.181)$

$(\mathrm{r}=0.099 \mathrm{p}<.001)$
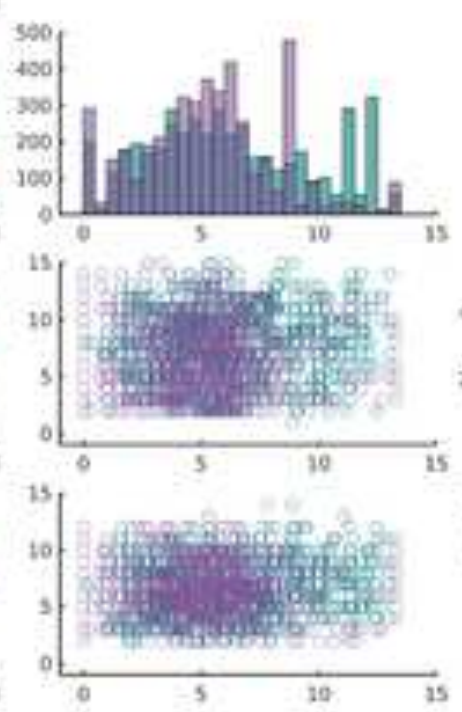

Index

$(x=0.07, \mathrm{p}<.001)$

$(r=-0.05, \mu=0.068)$

$(x=-0.09, p<.001)$
$r=-0.10, p<.001)$

$\{r=-0.15, p<.901)$ $(\mathrm{r}=-0.18, \mathrm{f}<001)$
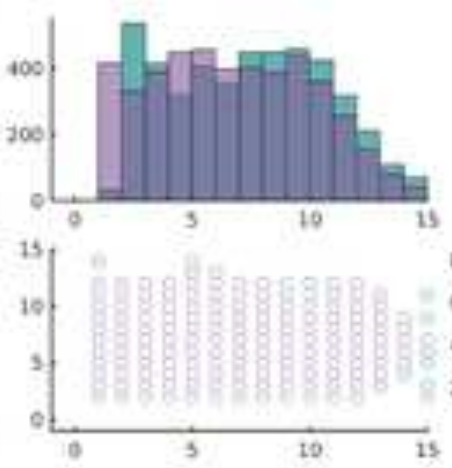

$(r=0.39, p<.001)$
$r=0.42, p<.1001\}$

Length

$(z=-0.52, \mathrm{p}<.001)$

$(r=-0.52, p<.001)$

$(p=-0.01, p=0.430)$

$\left\{\begin{array}{l}r=0.15, p<.001) \\ r=-0.04 \mathrm{p}=0.008\end{array}\right.$

$r=0.00, p=1.060)$

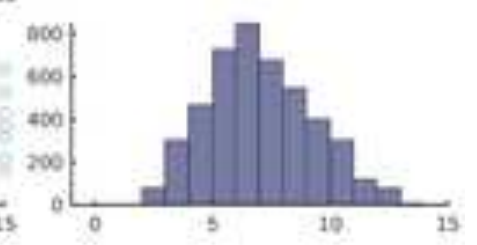


A

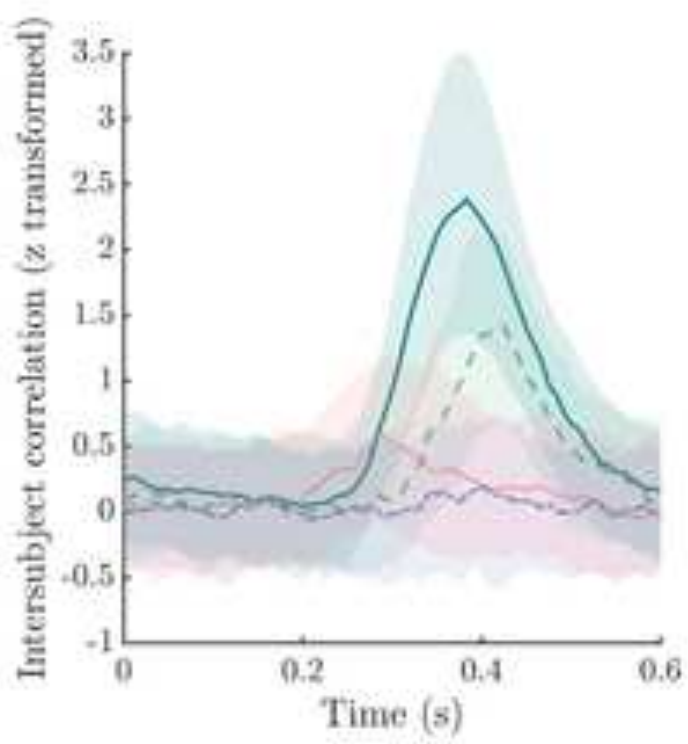

B

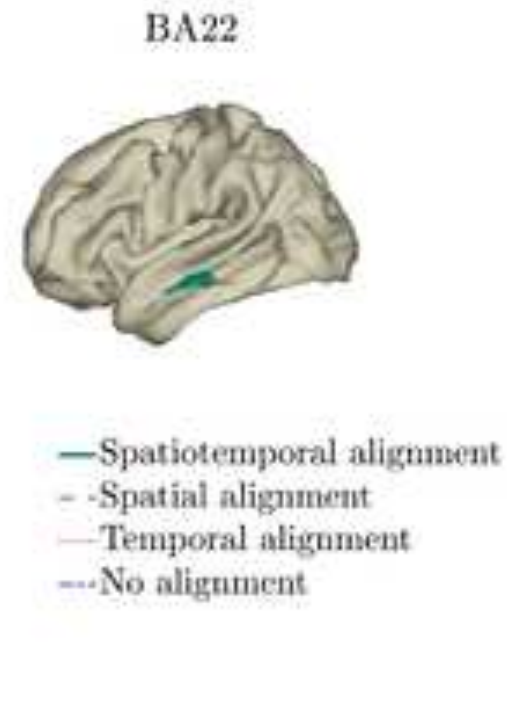

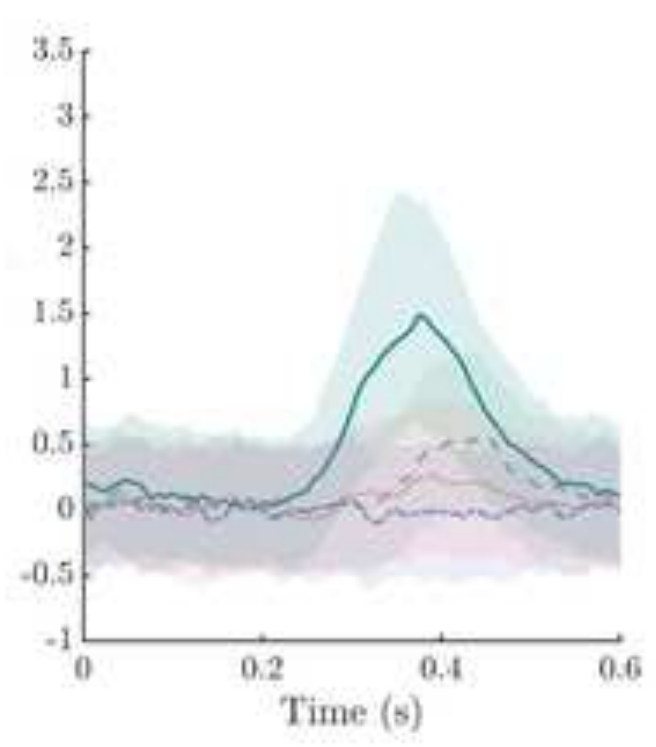

-Spatiotemporal aligament

- Spatinl aligmment

- Temporal alignment

-No alignment 


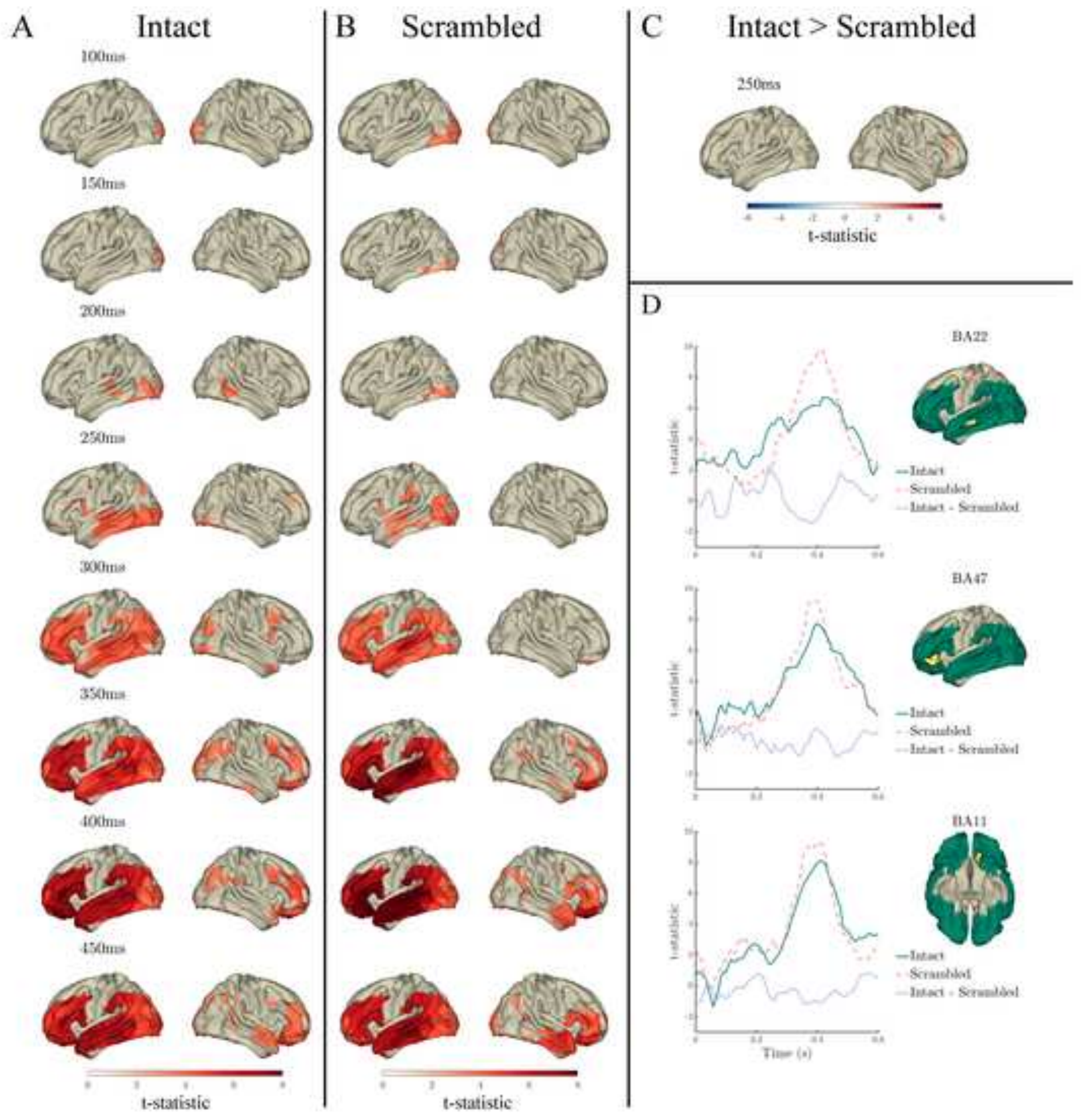




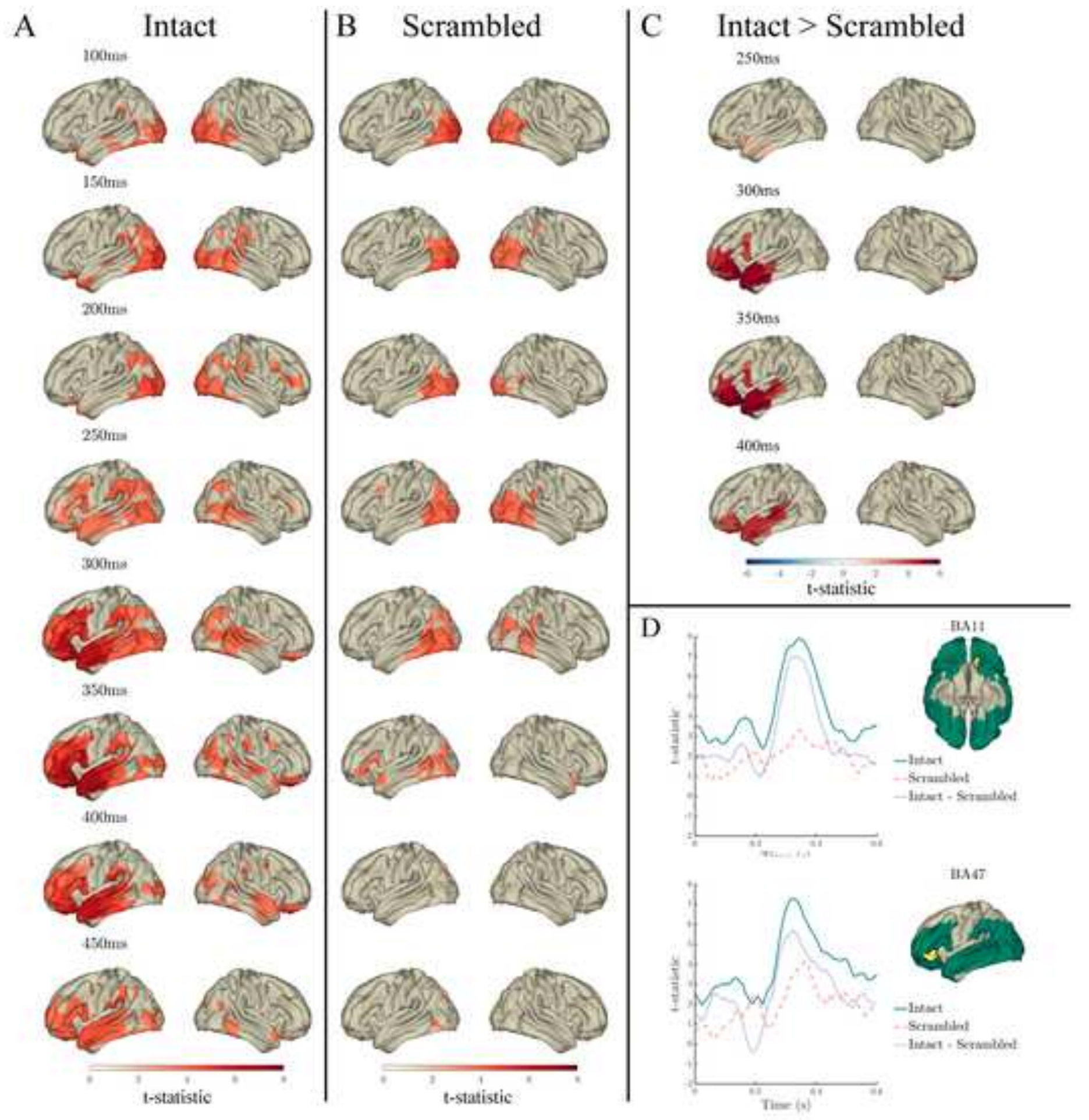




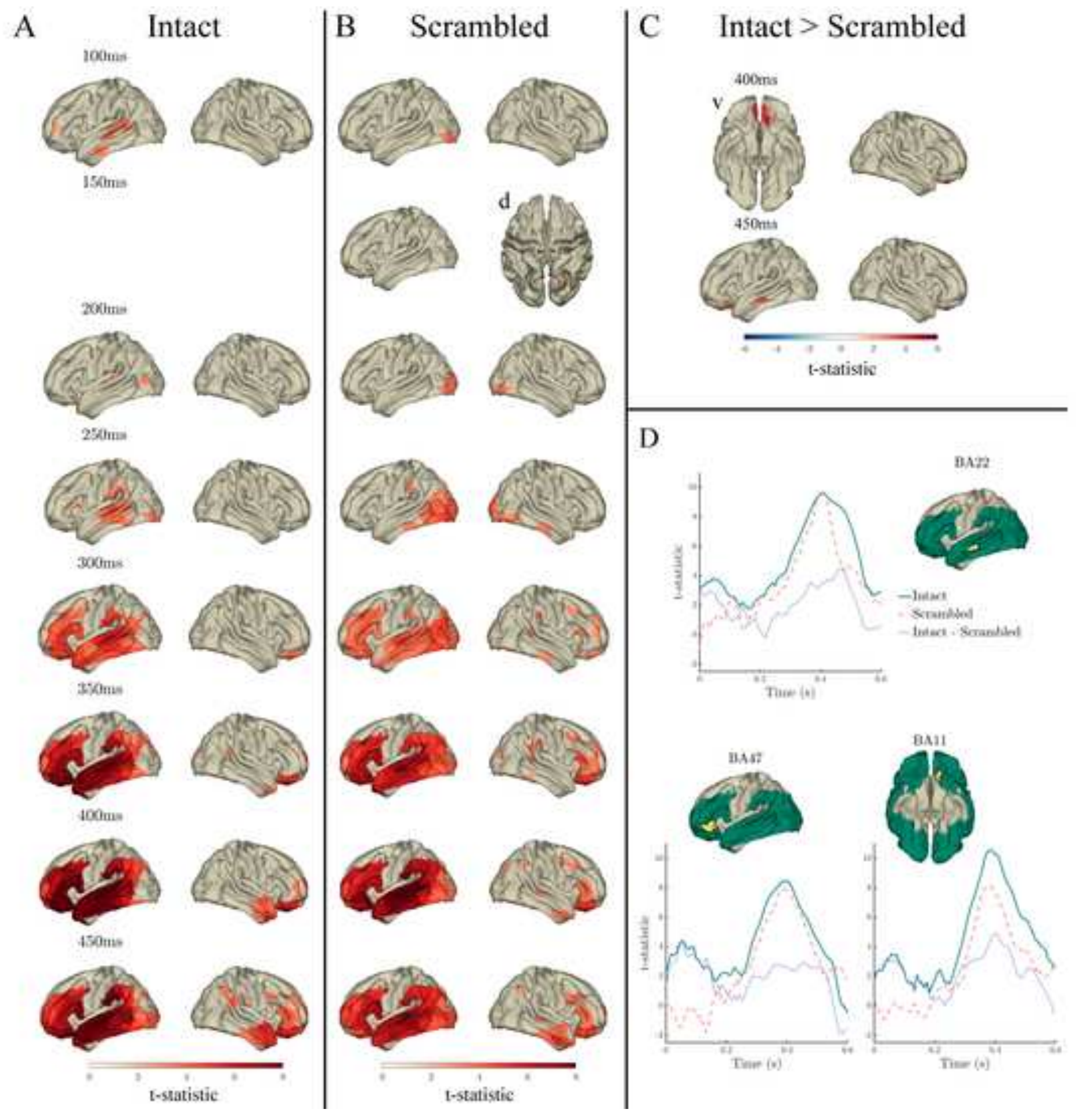


A
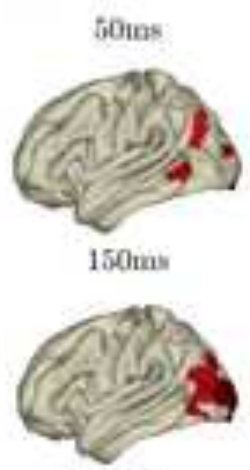

250 me

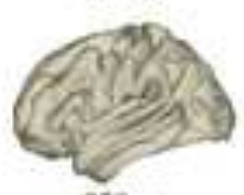

350 mes

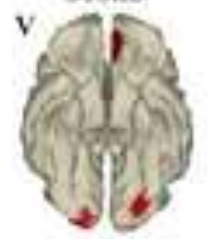

450ais:

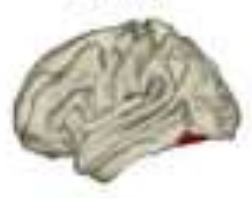

Intact
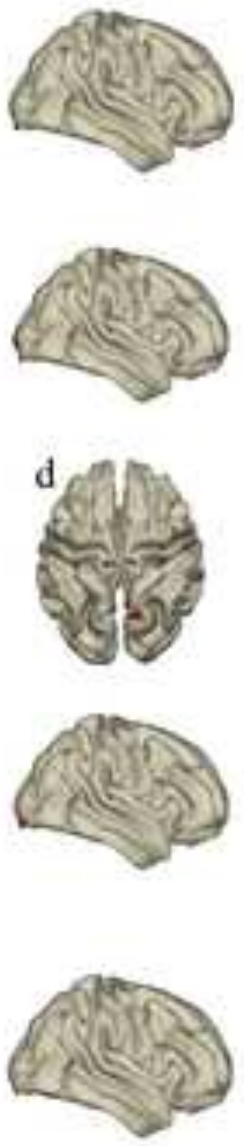

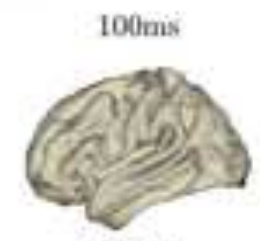

200 ma

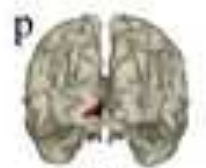

30thrs

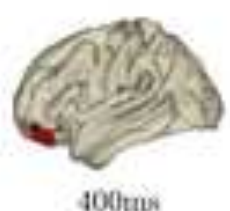

400 tas
B

Scrambled
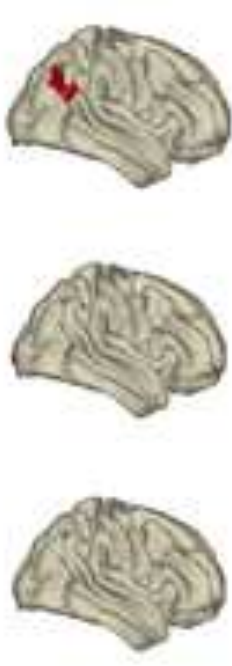

C Intact $>$ Scrambled
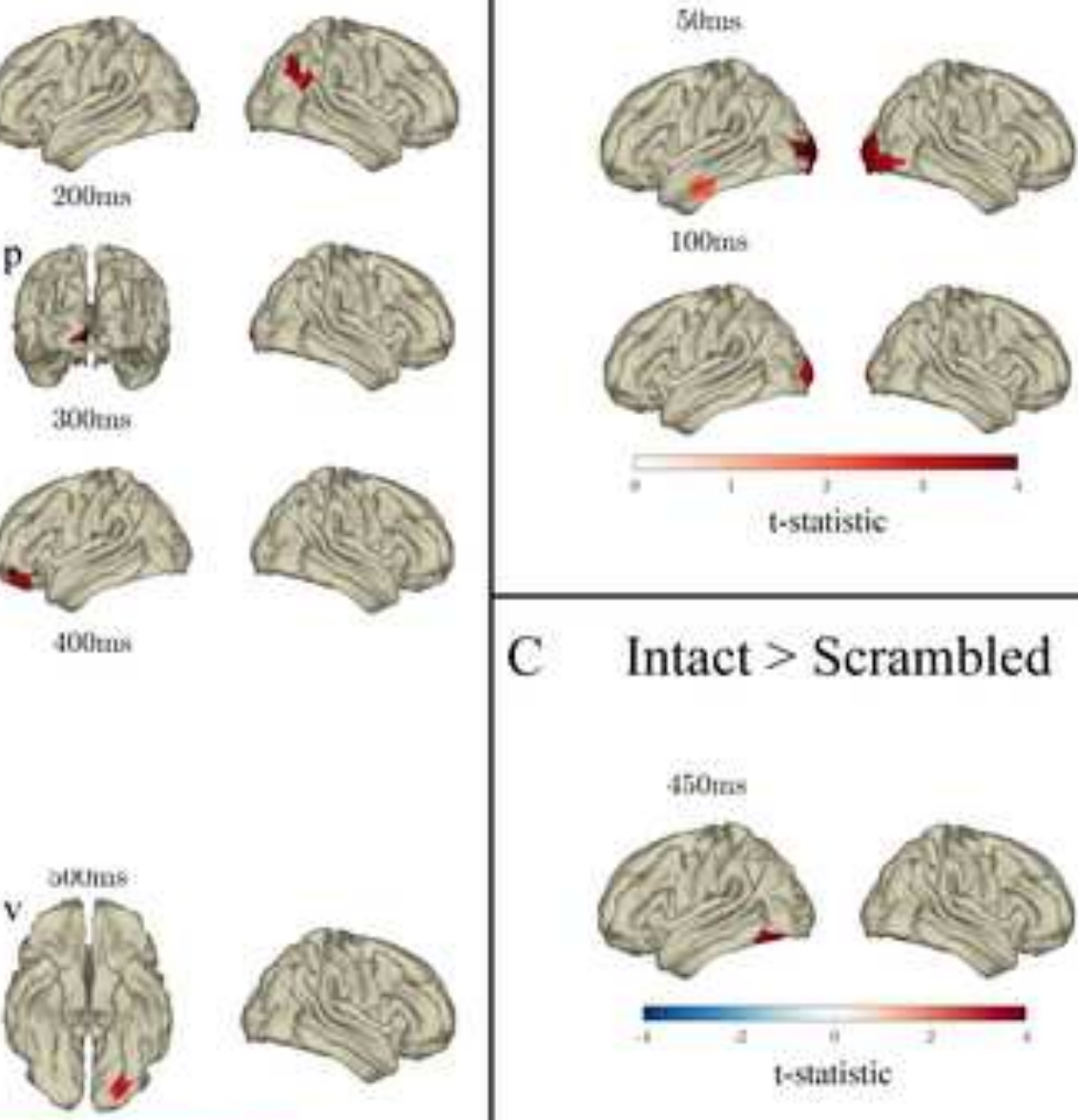

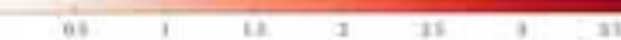

f-statistic

D
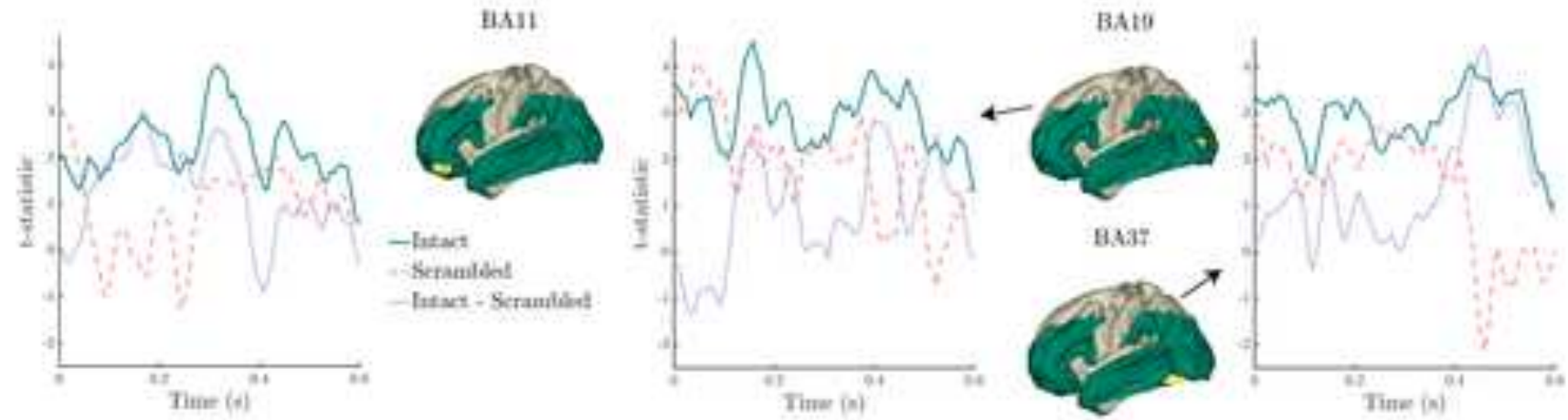


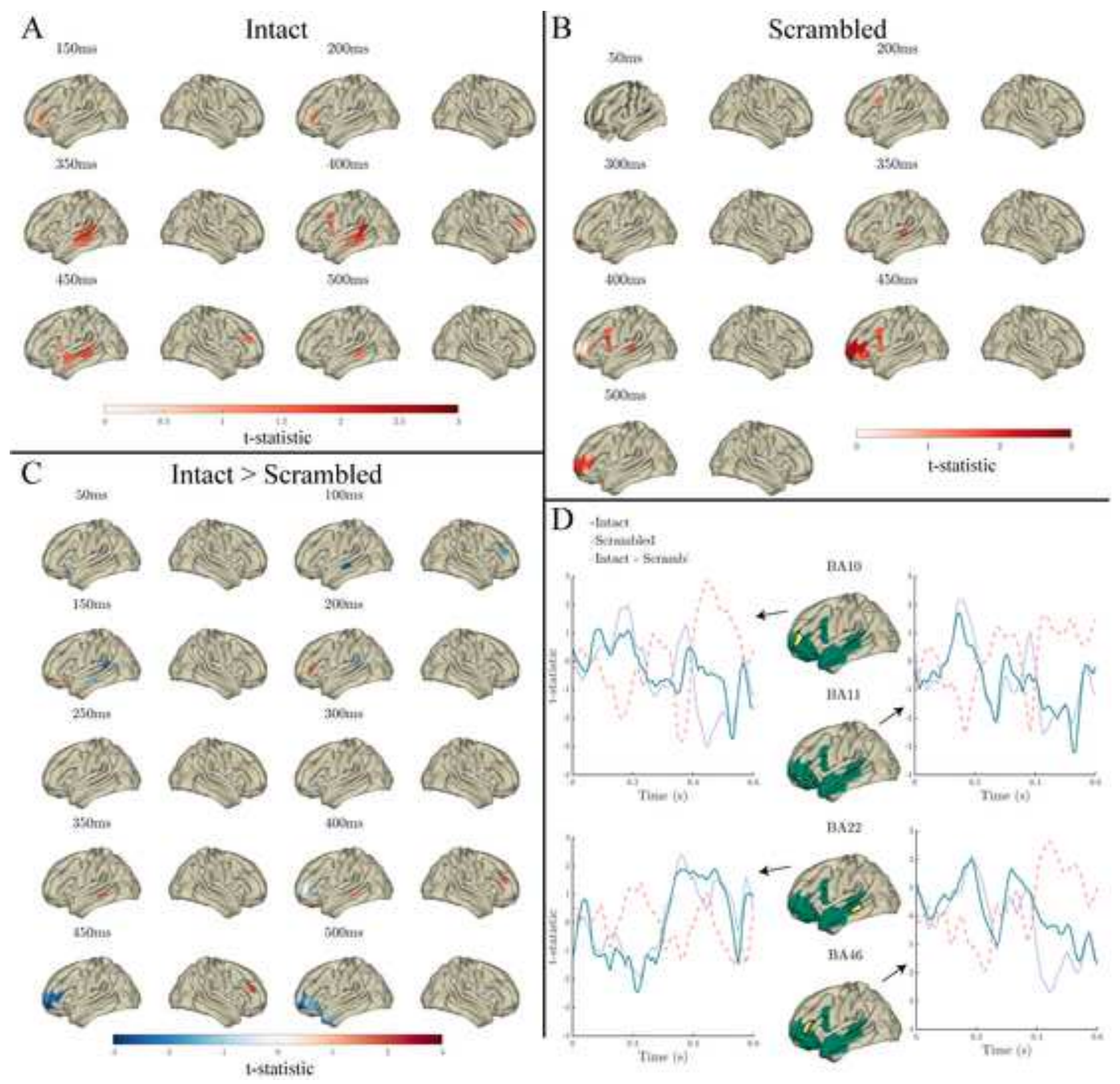


A

$250 \mathrm{~ms}$

Intact
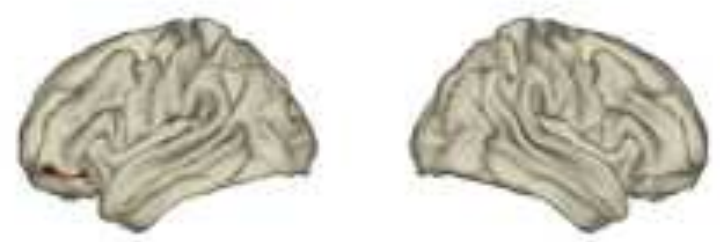

$350 \mathrm{~ms}$
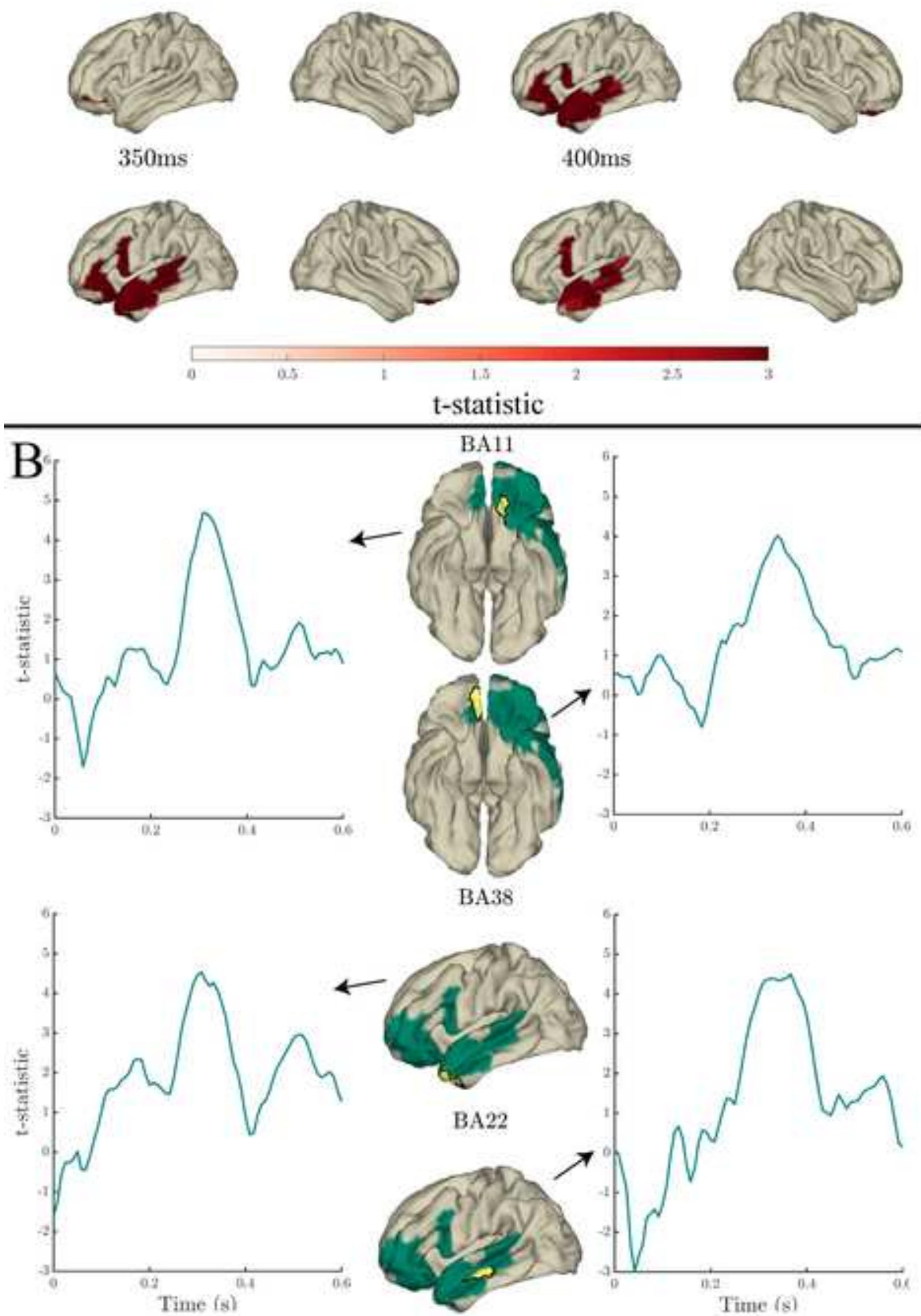

BA38
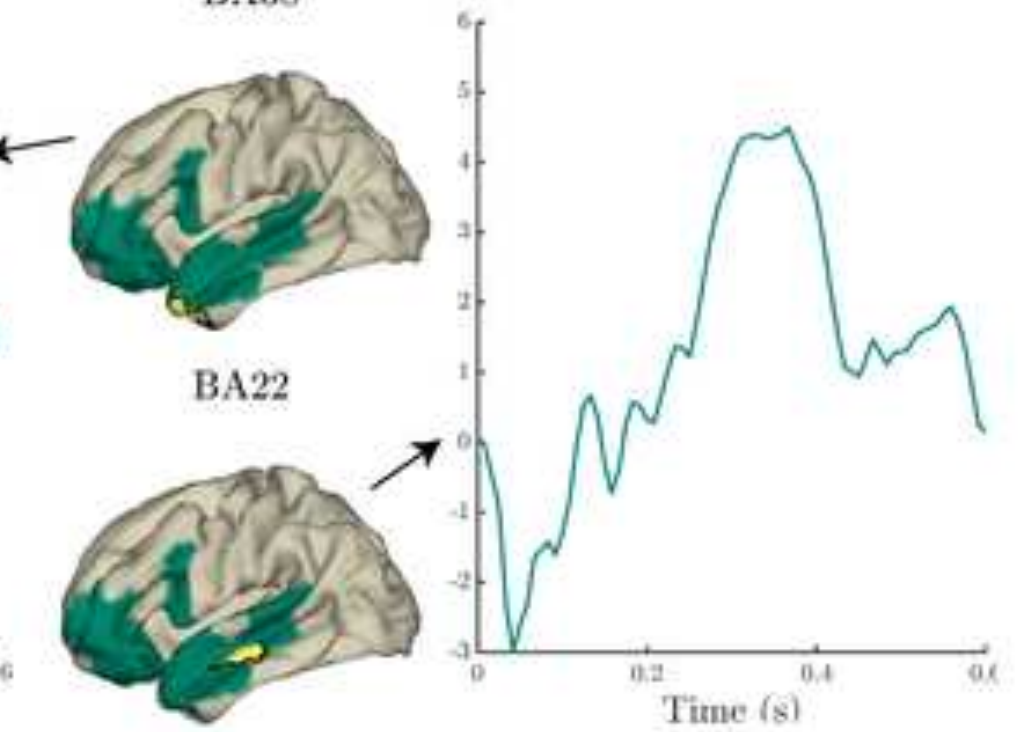


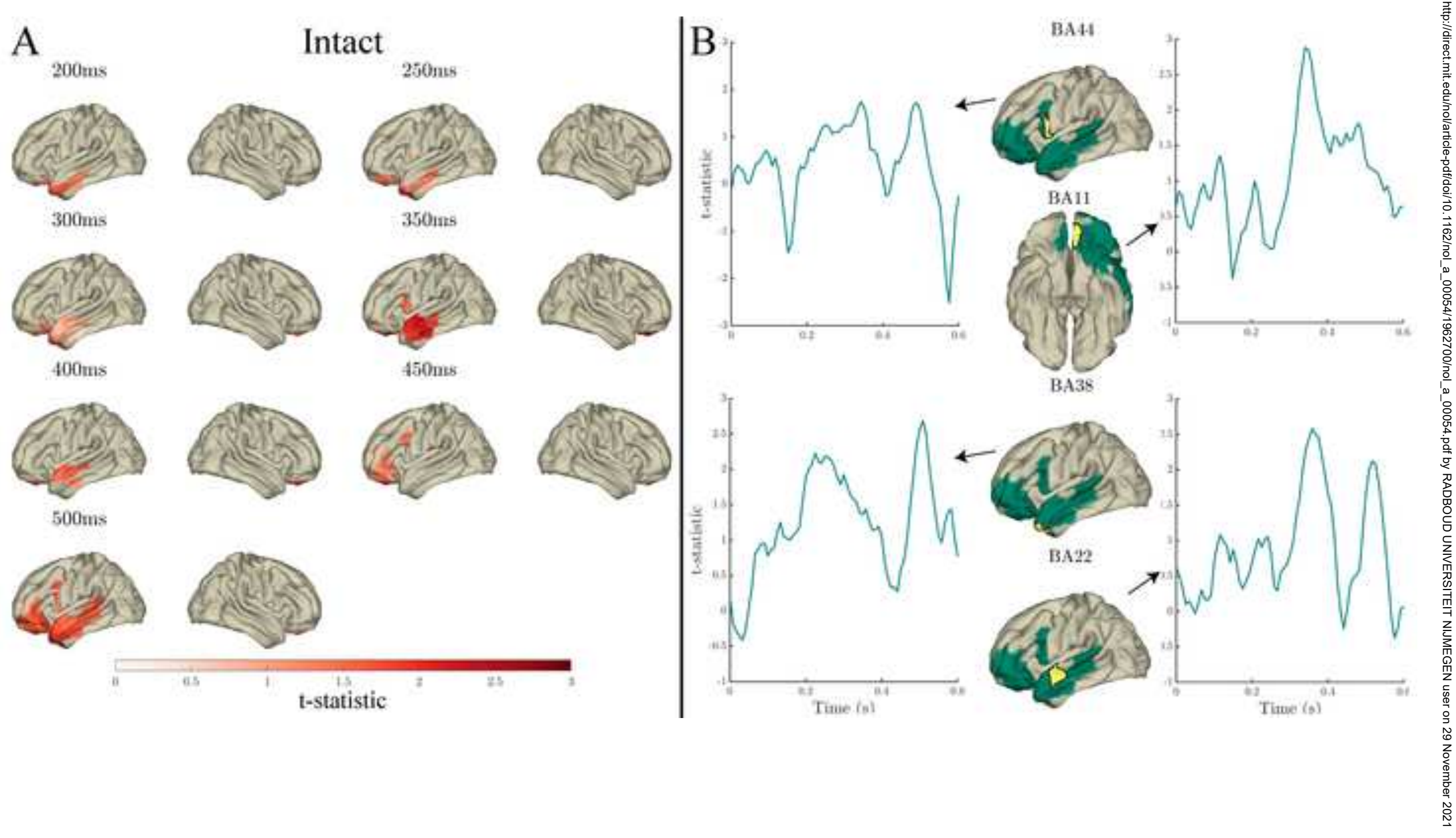

